\title{
Dislocations in Grain Boundary Regions: The Origin of Heterogeneous Microstrains in Nanocrystalline Materials
}

\author{
ZHENBO ZHANG, ÉVA ÓDOR, DIANA FARKAS, BERTALAN JÓNI, \\ GÁBOR RIBÁRIK, GÉZA TICHY, SREE-HARSHA NANDAM, JULIA IVANISENKO, \\ MICHAEL PREUSS, and TAMÁS UNGÁR
}

\begin{abstract}
Nanocrystalline materials reveal excellent mechanical properties but the mechanism by which they deform is still debated. X-ray line broadening indicates the presence of large heterogeneous strains even when the average grain size is smaller than $10 \mathrm{~nm}$. Although the primary sources of heterogeneous strains are dislocations, their direct observation in nanocrystalline materials is challenging. In order to identify the source of heterogeneous strains in nanocrystalline materials, we prepared $\mathrm{Pd}-10$ pct $\mathrm{Au}$ specimens by inert gas condensation and applied high-pressure torsion (HPT) up to $\gamma \cong 21$. High-resolution transmission electron microscopy (HRTEM) and molecular dynamic (MD) simulations are used to investigate the dislocation structure in the grain interiors and in the grain boundary (GB) regions in the as-prepared and HPT-deformed specimens. Our results show that most of the GBs contain lattice dislocations with high densities. The average dislocation densities determined by HRTEM and MD simulation are in good correlation with the values provided by X-ray line profile analysis. Strain distribution determined by MD simulation is shown to follow the Krivoglaz-Wilkens strain function of dislocations. Experiments, MD simulations, and theoretical analysis all prove that the sources of strain broadening in X-ray diffraction of nanocrystalline materials are lattice dislocations in the GB region. The results are discussed in terms of misfit dislocations emanating in the GB regions reducing elastic strain compatibility. The results provide fundamental new insight for understanding the role of GBs in plastic deformation in both nanograin and coarse grain materials of any grain size.
\end{abstract}

https://doi.org/10.1007/s11661-019-05492-7

(C) The Author(s) 2019

\section{INTRODUCTION}

THE role of dislocations in nanocrystalline solids has been an issue of discussion ever since nanocrystalline solids have become a hot topic for application and research. ${ }^{[1-6]}$ There is almost general consensus that when the average grain size is around $20 \mathrm{~nm}$ or smaller

ZHENBO ZHANG and MICHAEL PREUSS are with the School of Materials, University of Manchester, Oxford Road, Manchester M13 9PL, UK. Contact e-mail: ungar@ludens.elte.hu ÉVA ÓDOR, BERTALAN JÓNI, GÁBOR RIBÁRIK, and GÉZA TICHY are with the Department of Materials Physics, Eötvös Loránd University Budapest, PO Box 32, 1518 Budapest, Hungary. DIANA FARKAS is with the Department of Materials Science and Engineering, Virginia Tech, Blacksburg, VA 24060. SREE-HARSHA NANDAM and JULIA IVANISENKO are with the Institute for Nanotechnology, Karlsruhe Institute for Technology, 76021 Karlsruhe, Germany. TAMÁS UNGÁR is with the School of Materials, University of Manchester and also with the Department of Materials Science and Engineering, Virginia Tech.

Manuscript submitted April 15, 2019.

Article published online November 6, 2019 the grain interiors are free of dislocations. ${ }^{[2-4,6-10]}$ Obtaining evidence of grain interior structures with a grain size in the range of $20 \mathrm{~nm}$ or smaller is very difficult to obtain by Transmission Electron Microscopy (TEM), see for example the following figures from the literature: Figs. $3 \mathrm{a}$ and $3 \mathrm{~b}$ in Reference 11, Fig. 1 in Reference 12, and Fig. 3a in Reference 13. Though the TEM images in the work by Zhang et al. ${ }^{[13]}$ do indicate strain, the resolution is not sufficient to conclude on its origin. Dislocation activity in nanocrystalline materials has been widely investigated by molecular dynamic (MD) simulations. ${ }^{[2-4,7-10,14]}$ In many cases, MD simulations predict that grain boundaries (GBs) emit partial dislocations pulling stacking faults or twin boundaries decorating grain interiors at the end of straining. ${ }^{[2,3,8,10,12]}$ Line profile analysis of X-ray diffraction patterns reveals large microstrains in nanocrystalline materials. ${ }^{[4,5,7-9,15-18]}$ Markmann et al. ${ }^{[4,8]}$ evaluated the full width at half maxima (FWHM) of diffraction patterns of nanocrystalline $\mathrm{Pd}^{[19]}$ along with the FWHM of a computer-generated diffraction pattern of an 
MD-simulated specimen of the same material. Figure 1 in Reference 4 shows that the measured and computer-generated diffraction patterns are identical. The modified Williamson-Hall plots ${ }^{[20]}$ of the FWHM and the integral breadths of the computer-generated diffraction patterns have large positive slopes indicating the presence of significant microstrains. Both the real and the MD-simulated nanocrystalline Pd specimens have grain sizes of about $10 \mathrm{~nm}$. It was concluded that the microstrains in both the MD-simulated and experimentally measured nanocrystalline specimens are similar to those found in plastically deformed fcc metals, even though no specific lattice defects were introduced during the simulation process. ${ }^{[4]}$ It was further concluded in Reference 6 that the presence of microstrains, i.e., the positive slope in the modified Williamson-Hall plots in Fig. 2 in Reference 4, cannot be considered as evidence for the presence of dislocations. MD simulations of the GB regions, shown in Figs. 3 and 4 in Reference 6, revealed long-range correlated displacement fields extending well into the grain interiors; however, they were not directly correlated with any specific source of these strain fields. It is noteworthy that in this work (Fig. 3 in Reference 6) the GB regions were left blank with the atomic positions missing in the MD simulations. Due to the missing evidence of any specific lattice defects in the GB regions, it was concluded in Reference 4 that, though X-ray line broadening arises from long-range displacement fields emanating from the GBs, diffraction-based strategies for inferring the dislocation density in ultrafine-grained metals do not necessarily apply to nanocrystalline materials.

The physical and mechanical properties of nanocrystalline materials strongly depend on the nature and character of grain boundaries. MD simulation 'experiments' of Stukowski et al. ${ }^{[6]}$ indicate that substantial heterogeneous microstrains are potentially associated with grain boundaries or grain boundary regions. Large strain broadening in X-ray line profiles are in global correlation with these simulation "experiments. ${ }^{,[4,5,7,8]}$ In order to understand the mechanical behavior of nanograin materials, it is of imminent importance to find the source of heterogeneous microstrains associated with grain boundaries. For this aim, we prepared nanocrystalline Pd-10 at. pet Au alloy specimens by the method of inert gas condensation with an average initial grain size of about $12 \mathrm{~nm}$. One of the specimens was plastically deformed by high-pressure-torsion (HPT) up to a plastic shear strain of about $\gamma \cong 21$. The global dislocation density was determined by X-ray line profile analysis, whereas the local dislocation structure was obtained by High-Resolution Transmission Electron Microscopy (HRTEM). The dislocation structure of the material was also modeled by MD simulations. Both HRTEM and MD simulations provide direct evidence for dislocations in the GB regions, while the grain interiors of the nanocrystalline grains remain free of dislocations. The dislocation densities determined by HRTEM and MD-simulated images prove to be in good correlation with the values of the global dislocation densities given by $\mathrm{X}$-ray line profile analysis. The experimental and simulation results provide evidence of the presence of lattice dislocations in GB regions. These dislocations are the source of large heterogeneous microstrains and the corresponding substantial strain broadening in X-ray diffraction patterns. We show that the distortion distribution determined by MD simulation 'experiments' in Reference 6 can be well described by the Krivoglaz-Wilkens ${ }^{[21-23]}$ strain function typical when the source of heterogeneous strains are dislocations. Our HRTEM experiments along with MD simulations and the distortion distribution data in Reference 6 provide strong indications that the sources of strain broadening in X-ray diffraction patterns of nanocrystalline materials are overwhelmingly lattice dislocations in GB regions.

\section{EXPERIMENTAL}

\section{A. Materials}

Nanocrystalline Pd-10 at. pct Au powder was produced by inert gas condensation ${ }^{[24]}$ using a $10^{-7}$ mbar base pressure vacuum system and thermal evaporation of 99.95 pet purity $\mathrm{Au}$ and $\mathrm{Pd}$ in a 1 mbar $\mathrm{He}$ atmosphere. The powder was consolidated in situ at a pressure of $2 \mathrm{GPa}$ to obtain disk-shaped specimens with a diameter of $8 \mathrm{~mm}$ and thickness, $t=0.263 \mathrm{~mm}$. The nanocrystalline $\mathrm{Au}-10$ at. pct $\mathrm{Pd}$ samples exhibit a stable grain size even at temperatures well above room temperature. ${ }^{[11]}$ Even nanocrystalline pure Au shows stable grains size up to about $770 \mathrm{~K}$ and the grain size stability in nanocrystalline materials increases with alloying. ${ }^{[25]}$ The microstructure was studied in two initially identical specimens. One of the specimens was investigated in the as-prepared state and the other after deformation by high-pressure-torsion (HPT) of $1 / 4$ $(\chi=90 \mathrm{deg})$ rotation at a constant speed of 2 rotations/minute at a pressure of $p=6 \mathrm{GPa}$ in a custom-built computer-controlled HPT device (W. Klement $\mathrm{GmbH}$, Lang, Austria). The shear strain was calculated as $\gamma=\chi \mathrm{r} / t$ and it was $\gamma_{1 / 4 \mathrm{r}} \cong 7$ and $\gamma_{1 \mathrm{r}} \cong 21$ at third radius and close to the edge, respectively.

\section{B. X-ray Diffraction Experiments}

$\mathrm{X}$-ray diffraction experiments were carried out in a special high-resolution double crystal diffractometer with wavelength compensation dedicated to line profile analysis. It was built on the principles described in References 26-28. A plane Ge (220) primary monochromator operated at the $\mathrm{Cu} \mathrm{K} \alpha$ fine-focus rotating copper anode (Rigaku, RA-MultiMax9) at $40 \mathrm{kV}$ and 100 $\mathrm{mA} \cdot{ }^{[15]} \mathrm{A}$ narrow slit in front of the monochromator was adjusted to eliminate $\mathrm{CuK} \alpha_{2}$ radiation. After the monochromator, a second slit of $0.2 \times 1.0 \mathrm{~mm}^{2}$, close to the specimen, blocked parasitic scattering from the monochromator and reduced beam divergence normal to the incidence plane. The distance between the X-ray source and the specimen was $560 \mathrm{~mm}$. This setup provides a monochromatic and almost parallel beam with a divergence less than about 0.025 deg in the plane of incidence. The footprint of the beam on the specimen 
was about $0.2 \times 1.0 \mathrm{~mm}^{2}$. Since, in the present case, the narrowest diffraction peaks in all measured diffraction patterns were at least one order of magnitude broader than the instrumental breadth of $0.057 \mathrm{deg}$, there was no need for instrumental corrections. The diffracted beam was recorded using three curved image plates (IP) with a linear spatial resolution of $50 \mu \mathrm{m}$. The IPs were positioned at a distance of $300 \mathrm{~mm}$ from the stationary specimen, covering the $2 \theta$ angular range from 30 to 153 deg. The X-ray beam was positioned on the specimen surface by using a low depth-resolution microscope coupled to a TV screen. X-ray diffraction measurements were carried at about the center, at one-third and two-third of the radius and close to the edge of the samples as shown in Figure 1(a). Diffraction images recorded by three curved image plates from the center and $2 / 3 R$ position of the HPT-deformed specimen are shown in Figure 1(b). The diffraction patterns were obtained by integrating the intensity distributions along the Debye-Scherrer arcs on the image plates. Only the central parts of the arcs were used for integration where the geometrical spreading of Debye-Scherrer arcs does not affect line broadening. The diffraction patterns of the as-received inert gas-condensed specimen and the HPT-deformed specimen at 0.25 rotation, measured close to the edge, are shown in Figure 1(c). The logarithmic intensity scale is used to better see the shape of the peaks in the entire intensity range. A closer inspection reveals that the peaks narrow after deformation in correlation with both grain growth and reduction in dislocation density. In order to see the difference between measured and CMWP-calculated intensity distributions, the as-received pattern is shown in Figure 1(d) with linear intensity scales.

\section{Evaluation of the X-ray Diffraction Patterns}

The diffraction patterns are evaluated by using the convolutional multiple whole profile (CMWP) procedure. ${ }^{[29,30]}$ The method is based on physically well-established profile functions theoretically calculated for different specific lattice defects, in particular for (i) coherently scattering domain size, ${ }^{31]}$ (ii) dislocations, ${ }^{[21-23]}$ and (iii) various planar defects. ${ }^{[32,33]}$ The size profile function is given by the median, $\mathrm{m}$, and the variance, $\sigma$, for the coherently scattering domain size. The strain profile function is given by the density, $\rho$, the average contrast factors, $\bar{C}$, and the arrangement parameter, $\mathrm{M}$, of dislocations. The profile function of planar defects is given as the sum of symmetric and

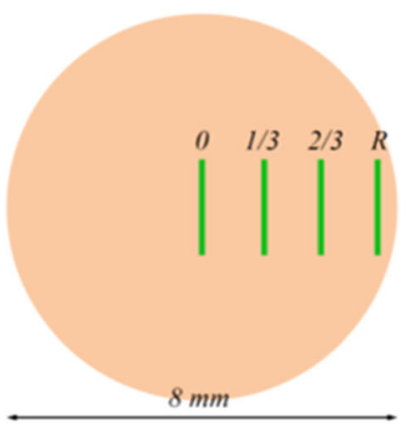

(a)

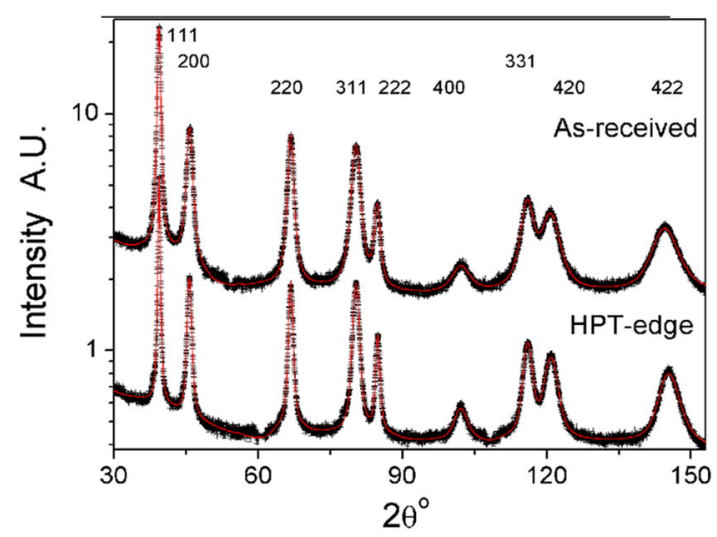

(c)

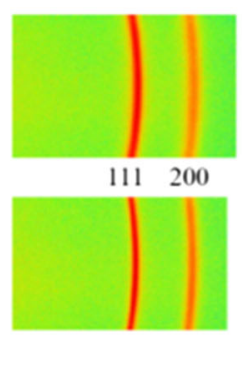

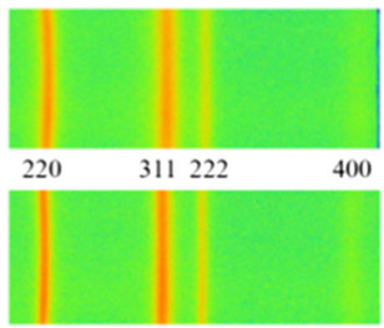

(b)

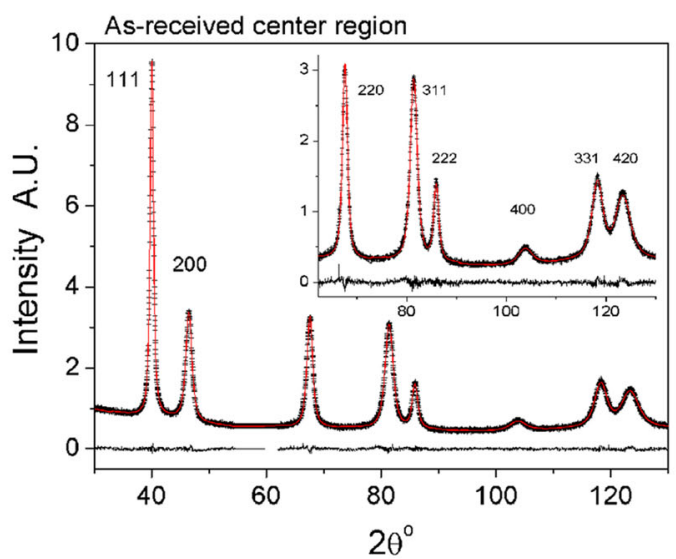

(d)

Fig. 1-X-ray diffraction results: (a) schematic image of the HPT-deformed PdAu diskette showing the footprints of the X-ray beam in true relative scale; $(b)$ image plate records from the as-received and $2 / 3 R$ position of the HPT-deformed specimen, where $R$ is the radius; $(c)$ diffraction patterns obtained by integrating the central region of the image plate readout from the as-received and the edge of the HPT specimen in logarithmic intensity scale. Crosses are the measured and red line the CMWP-calculated patterns. $(d)$ Same as the as-received patterns in $(c)$ with linear intensity scale. The difference between the measured and CMWP-calculated patterns is shown in the lower part of the figure. The inset is the enlarged part in the higher angle section of the pattern. 
antisymmetric Lorentz functions $v s$ the density of planar faults. ${ }^{[32-37]}$ The calculated and measured diffraction patterns are matched to each other by adjusting the physical parameters listed above. Instrumental effects, if necessary, are also convoluted to the physical profiles. The background is determined either manually or by a background fitting procedure. Matching of the calculated and measured patterns is done by combining the Marquard-Levenberg analytical least-squares and a Monte Carlo statistical optimization procedure. ${ }^{[38]}$ The two procedures are applied iteratively in order to obtain the global optimum for the physical parameters: the crystallite size distribution parameters, i.e., the median, $m$, and variance, $\sigma$, of the log-normal size distribution function, the dislocation density and arrangement parameter, $\rho$ and $M$, and the planar defect density, $\beta$, respectively. ${ }^{[38]}$

\section{Size broadening}

Size broadening is produced by small coherently scattering domains ${ }^{[31]}$ also called crystallites. The size distribution of the coherently scattering domains is taken into account by assuming log-normal size distribution function, $f(x)$, given by the median, $\mathrm{m}$, and the variance, $\sigma$ :

$$
f(x)=\frac{1}{\sqrt{2 \pi \sigma}} \frac{1}{x} \exp \left\{-\frac{[\ln (x / m)]^{2}}{2 \sigma^{2}}\right\} .
$$

The size profile is given by convoluting the size function of coherently scattering domains and the log-normal size distribution function ${ }^{[29,30,38]}$.

$$
\operatorname{IS}(\mathrm{s})=\int_{0}^{\infty} x \frac{\sin ^{2}(x s)}{(s)^{2}} \operatorname{erfc}\left[\frac{\ln (x / m)}{\sqrt{2}}\right] \mathrm{d} x,
$$

where erfc is the complementary error function. It can be shown that the best match between TEM and $\mathrm{X}$-ray size is provided by the area-weighted mean crystallite size ${ }^{[29,30,38,39] \text {. }}$

$$
\langle x\rangle_{\text {area }}=m \times \exp \left(2.5 \times \sigma^{2}\right) .
$$

When the crystallites are equiaxed size broadening is isotropic, i.e., it is independent of scattering order. If the crystallites are oblate or elongated, then size broadening becomes $h k l$ dependent, i.e., anisotropic as a function of diffraction order. ${ }^{[40,41]}$ Since the $h k l$ dependence of anisotropic size broadening is different from the $h k l$ dependence of strain anisotropy, the two anisotropies can be distinguished from each other. ${ }^{[29,30,40,41]}$

In a comprehensive analysis of crystallite size and grain size obtained by X-ray line broadening and TEM in the same specimens, it was shown that, when the TEM grain size becomes smaller than a few hundred nanometers, then the X-ray crystallite size tends to be identical with the TEM grain size. ${ }^{[39]}$ The physical reason for this is that in large grain size samples the $\mathrm{X}$-ray crystallite size provides the sub-grain size which is usually smaller than the grain size given by TEM. If, however, the grain size becomes of the order of a few hundred nanometers or smaller, then such grains are usually not divided further by smaller sub-grains.

\section{Strain broadening}

The Fourier transform of the strain profile can be written as ${ }^{[42]}$

$$
A_{h k l}^{\mathrm{D}}(L) \cong \exp \left[-2 \pi^{2} g^{2} L^{2}\left\langle\varepsilon_{g, L}^{2}\right\rangle\right]
$$

where $g$ is the absolute value of the diffraction vector, $L$ is the Fourier variable, and $\left\langle\varepsilon_{g, L}^{2}\right\rangle$ is the mean square strain. For dislocated crystals, the mean square strain was elaborated by Krivoglaz, ${ }^{[21,22]}$ Wilkens, ${ }^{[23]}$ and Groma et al. ${ }^{[43]}$.

$$
\left\langle\varepsilon_{g, L}^{2}\right\rangle \frac{\rho \bar{C} b^{2}}{4 \pi} f(\eta)
$$

where $\rho$ and $b$ are the density and Burgers vector of dislocations, $\bar{C}$ is the average dislocation contrast factor and $f(\eta)$ is the strain function. The function $f(\eta)$ describes the $L$ dependence of the mean square strain with $\eta=L / R_{\mathrm{e}}$, where $R_{\mathrm{e}}$ is the effective outer cut-off radius of dislocations. The physical meaning of $R_{\mathrm{e}}$ here is the same as in the elastic stored energy of dislocations. ${ }^{[44-46]} R_{\mathrm{e}}$ is rationalized as the dipole character of dislocation arrangements introducing the dimensionless arrangement parameter $M=R_{\mathrm{e}} \sqrt{\rho} .^{[23]}$ If $M$ is smaller or larger than about unity, the dipole character of dislocations is stronger or weaker, respectively. For very strong dipole character of dislocation arrangements, e.g., in the cell walls of persistent slip bands in high-cycle fatigued copper single crystals $M \cong 0.7 .^{[47]}$ For loosely distributed dislocations with weaker dipole character, e.g., in tensile-deformed copper single crystals $M \cong 2.3 .^{[48]} M$ correlates with the profile shape. ${ }^{[47]}$ For smaller or larger $M$ values, the tails of diffraction peaks become longer or shorter, respectively. ${ }^{[23,29,30]}$

Equation [5] shows that the mean square strain also depends on the contrast factors, $\bar{C}$, of dislocations. The physical reason for this is that strain broadening depends on the relative orientation between the Burgers and line vectors of dislocations and the diffraction vector, $\boldsymbol{b}, \boldsymbol{l}$, and $\boldsymbol{g}$, and the elastic constants, $c_{i j k l}$, of the material, ${ }^{[20-23,49-51]}$ making strain broadening $h \mathrm{kl}$ dependent. The effect is called strain anisotropy which can be taken into account by the dislocation contrast factor, $C=C\left(\boldsymbol{b}, \boldsymbol{g}, \boldsymbol{l}, c_{i j k l}\right)$. Strain anisotropy is well known in TEM where tilting of a specimen changes the contrast of dislocations. Strain anisotropy in X-ray diffraction and TEM has the same physical background. The contrast factor is a purely geometrical parameter and can be calculated theoretically for specific dislocations and $h k l$ values in any material. ${ }^{[50,52]}$ In a texture-free polycrystal or a powder specimen, the contrast factor can be averaged over the permutations of $h \mathrm{kls}$. For cubic systems, the $h k l$ dependence of the average contrast factors is ${ }^{[53]}$

$$
\bar{C}=\bar{C}_{h 00}\left(1-q H^{2}\right),
$$


where $\bar{C}_{h 00}$ is the average contrast factor of the $h 00$ reflections and $H^{2}=\left(h^{2} k^{2}+h^{2} l^{2}+k^{2} l^{2}\right) /\left(h^{2}+k^{2}+\right.$ $\left.k^{2}\right)^{2}$. The q parameter depends on the elastic constants of the crystal, and the type of dislocations, i.e., on the slip system and screw or edge character, respectively, and can be evaluated numerically. ${ }^{[50-53]}$

In the present $f c c$ crystal structure, the major operating slip system is $\{111\}\langle 110\rangle$. The contrast factors were evaluated for edge- and screw-type dislocations with $\langle 110\rangle$ Burgers vectors on $\{111\}$ slip planes. Dislocation types were analyzed in an MD-simulated nanocrystalline polycrystal sample of $f c c$ structure. ${ }^{[54]}$ It was shown that a large fraction of dislocations in the GB region are partials with Burgers vectors of 1/6[121]. Equation [2] shows that the dislocation density is scaled by $\bar{C} b^{2}$. The average contrast factors, $\bar{C}$, of the $1 / 2[110]$ and $1 / 6[121]$ Burgers vectors are the same for all hkl reflections within about 15 pct. $^{[50]}$ The major difference in the $\bar{C} b^{2}$ scaling factor is given by $b^{2}$. The ratio of the two scaling factors is $\bar{C} b_{[110]}^{2} / \bar{C} b_{[121]}^{2} \cong 3$. This has been taken into account in evaluating the dislocation density values listed in Tables I and III.

\section{Operation of the CMWP procedure}

The physically modeled diffraction patterns, $I^{\mathrm{PM}}(2 \theta)$, are the convolution of the physically modeled profile functions of crystallite size, $I_{h k l}^{\mathrm{S}}$, strain caused by dislocations, $I_{h k l}^{\mathrm{D}}$, planar defects, $I_{h k l}^{\mathrm{PD}},{ }^{[32,33]}$ and the $I_{h k l}^{\mathrm{Inst}}$, instrumental profiles. The background, BG, is added to the convoluted model profiles:

$$
I^{\mathrm{PM}}(2 \theta)=\sum_{h k l} I_{h k l}^{\mathrm{S}} \times I_{h k l}^{\mathrm{D}} \times I_{h k l}^{\mathrm{PD}} \times I_{h k l}^{\mathrm{Inst}}+B G .
$$

Two typical measured (crosses) and CMWP-calculated (red lines) patterns are shown in Figure 1(c). The values of the physical parameters are limited in the CMWP procedure in wide ranges of bounds which can be edited by the user ${ }^{[38]}$ The errors of the physical parameter values are determined in terms of the $p$ pct fractions of the weighted-sum-of-squared-residuals (WSSR), $\Delta \mathrm{WSSR}=\mathrm{WSSR}(1+p$ pct $)$, in the Monte Carlo algorithm after the last iteration step. ${ }^{[38]}$ Both the number of iteration steps and the value of $p$ can be edited by the user. In the present case, $p$ was set to $p=3.5$ pct. More details of the algorithms used in the CMWP procedure can be found in Reference 38.

\section{Elastic compatibility strains or stresses}

Compatibility stresses related to strain gradients can build up by geometrically necessary dislocations (GNDs). ${ }^{55-58]}$ It has been proved in a number of works that X-ray peak broadening catches both GNDs and statistically stored dislocations (SSD) together. For example, SSDs in dislocation cell walls along with GNDs producing long-range internal stress in tensile-deformed copper single crystals ${ }^{[59]}$ or GND-type misfit dislocations at the interfaces between $\gamma$ and $\gamma^{\prime}$ phases along with SSDs within the $\gamma$ channels in Ni-base superalloys $^{[60]}$ or GNDs at the interphase between martensite and austenite layers along with the SSDs

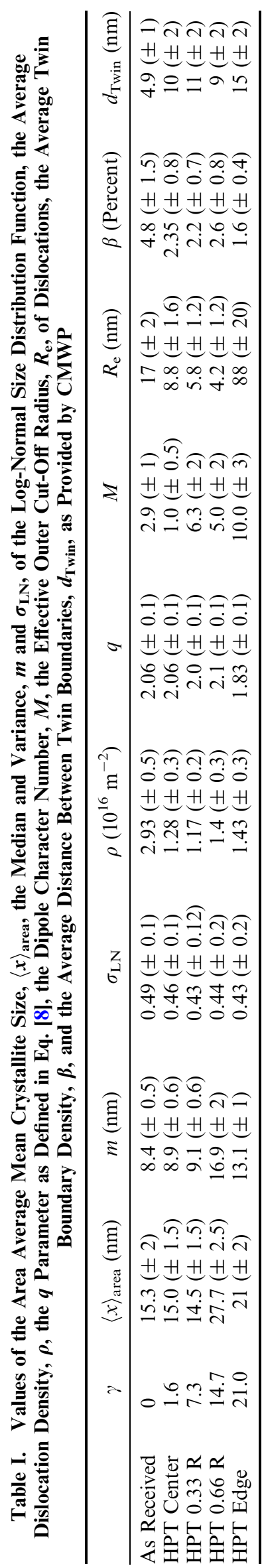

VOLUME 51A, JANUARY 2020-517 
within the laths in tensile-deformed martensite steels, ${ }^{[61]}$ all are captured together in peak broadening. Elastic compatibility strains or stresses (ECSs) can exist below the critical resolved shear stress (CRSS). ${ }^{[57,62,63]}$ If necessary, these can be treated in CMWP by an additional profile function convoluted to the other modeled profile functions in Eq. [5]. ${ }^{[38]}$

\section{Dimensional Classification of Lattice Defects}

Krivoglaz $^{[22]}$ showed that strain broadening of X-ray line profiles can only be caused by dislocations or linear-type lattice defects. Lattice defects can be sorted into three dimensional categories, (i) zero, (ii) one, and (iii) two dimensional, which are point defects, linear defects, i.e., dislocations or dislocation-like defects, and planar defects, i.e., twin boundaries, stacking faults, or grain boundaries, respectively. The strain fields are of short range, i.e., $\varepsilon \propto 1 / r^{2}$, long range, i.e., $\varepsilon \propto 1 / r$ and constant or homogeneous, respectively, where $r$ is the distance from the defects. Due to reciprocity between crystal and reciprocal space, the intensity distribution corresponding to the three defects categories will be (i) smoothly varying below the Bragg peaks, ${ }^{[64]}$ (ii) clustered around the fundamental Bragg reflections, ${ }^{[23]}$ and (iii) causing peak shifts, $c f$. References 32-37, respectively. The first is often called Huang scattering. ${ }^{[64]}$ Line broadening affects the Bragg peaks and is usually restricted to small, $\Delta K / K \leq 5 \times 10^{-2}$, ranges. ${ }^{[23,27,47]}$ According to the dimensional hierarchy of lattice defects introduced by Krivoglaz, ${ }^{[22]}$ strain broadening of diffraction peaks is an indirect indication of the presence of dislocations.

\section{E. Electron Microscopy Characterization}

$\mathrm{X}$-ray line profile analysis gives the global dislocation density in the specimen. In order to determine the location and arrangement of dislocations, we carried out HRTEM investigations. The microstructure and micro-texture of the samples before and after HPT were characterized by scanning electron microscopy (SEM) and TEM. Electron-transparent samples were prepared from the cross section of the samples by focused ion beam using an FEI Quanta 3D SEM electron microscope. In the case of the HPT-deformed specimen, the TEM foils were prepared from the regions about halfway between the center and the edge of the disks. TEM observation and high-resolution HRTEM imaging were conducted in an FEI F30 field emission gun TEM operated at $300 \mathrm{kV}$. Transmission Kikuchi Diffraction (TKD) technique was used for micro-texture analysis. TKD was carried out in an FEI Magellan 400 SEM equipped with an Oxford-Aztec system operated at $30 \mathrm{kV}$ with a probe current of 1.6 $\mathrm{nA}$. The sample was tilted to an angle of $20 \mathrm{deg}$ with respect to the electron beam. A step size of $2 \mathrm{~nm}$ was chosen, and the data were collected by Aztec and analyzed using the Channel5 software of Oxford Instruments. No appreciable texture was observed in the two investigated samples.

\section{F. Molecular Dynamics Simulation Methodology}

Grain interiors in nanocrystalline materials are usually found to be free from dislocations. ${ }^{[2-4,6-10]} \mathrm{X}$-ray line broadening, however, reveals large strain broadening in nanocrystalline materials ${ }^{[6]}$ the source of which has not been clarified yet. We carry out MD simulations to support HRTEM experiments in order to have more evidence of the nature and location of dislocations causing large strain broadening in nanocrystalline specimens in which the grain interior regions are free from dislocations. Digital samples of nanocrystalline pure Pd and pure $\mathrm{Au}$ were generated to mimic the experimental nanocrystalline samples. The technique for sample generation is based on a Voronoi construction ${ }^{65]}$ technique in three dimensions and randomly chosen grain orientations and centers. Periodicity was utilized in all three directions to avoid any effects of free surfaces. This technique has been used extensively to study the properties of nanocrystalline materials, and it implies general grain boundaries with no preference to any special misorientation or grain boundary planes. The samples generated were cubes of $20 \mathrm{~nm}$ side and contained 12 grains of average diameter $7.5 \mathrm{~nm}$. The total number of atoms in each simulation was 496,772. The samples were relaxed at $300 \mathrm{~K}$ for 100 ps using molecular dynamics and an Embedded Atom Method (EAM) interatomic potentials developed for $\mathrm{Au}$ and Pd. ${ }^{[66]}$ The molecular dynamics implementation used is that of LAMMPS. ${ }^{[67]}$ The relaxation was performed maintaining a constant zero pressure separately in all faces of the sample. This procedure resulted in relaxed grain boundary structures in a sample with no macroscopic stress in any of the spatial directions. Visualization of the digital samples was performed using OVITO. ${ }^{[68,69]}$ The primary goal of the visualization was the identification of any dislocations that were present in the relaxed sample, either inside the grains or as part of the grain boundary structure.

\section{RESULTS}

\section{A. Global Dislocation Densities in the Nanocrystalline Au-10 Pct Pd Specimens Determined by X-ray Line Profile Analysis}

X-ray diffraction measurements were carried on both the undeformed and the deformed specimens. In the sample deformed by HPT, strain increases from the center to the edge. With the fine footprint of the X-ray beam on the specimen of about $200 \times 1500 \mu \mathrm{m}$, X-ray diffraction patterns were measured as a function of distance from the center of the deformed specimen shown in Figure 1. This provides global dislocation densities as a function of shear strain shown in Figure 1(a). Close inspection of the patterns in Figure 1(c) reveals that the peaks narrow after HPT. The X-ray diffraction patterns were evaluated for the area average mean crystallite size, $\langle x\rangle_{\text {area, }}$ the average dislocation density, $\rho$, the average dislocation contrast factors, $\bar{C}$, the average twin boundary density, $\beta$, and the 


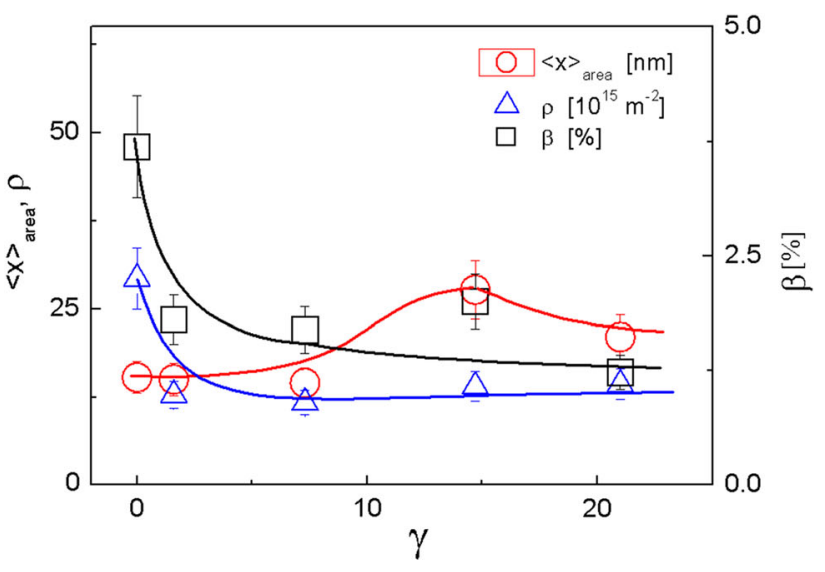

(a)

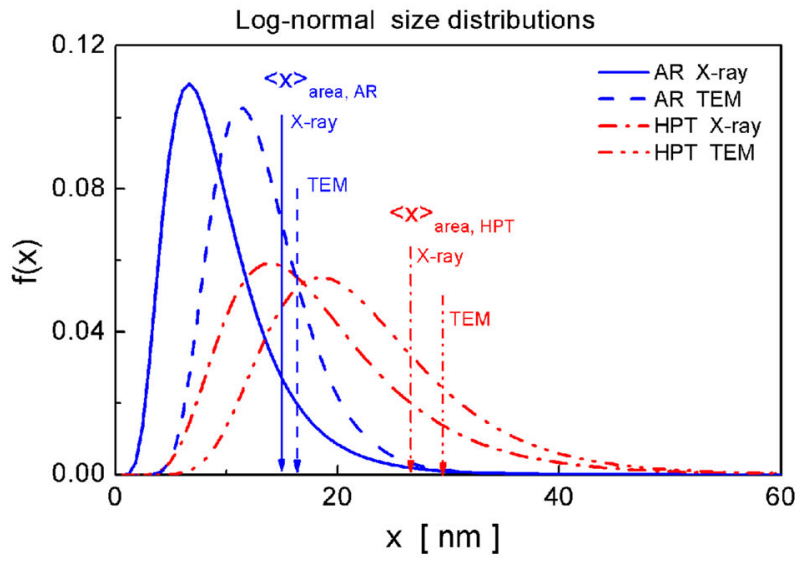

(b)

Fig. 2-X-ray diffraction analysis: (a) The area average mean crystallite size, $\langle x\rangle_{\text {area }}$ (open red circles), the dislocation density, $\rho$ (open blue triangles), and the twin density, $\beta$ (open black rectangles). The lines through the data points are only to guide the eye. (b) Log-normal size distribution functions of crystallite size for the as-received and HPT-deformed specimen determined either by X-rays (blue solid and red dash-dot lines) or from dark-field TEM micrographs (blue dash and red dash double-dot lines). The X-ray data correspond to the $2 / 3 R$ region in the disk. The vertical arrows indicate the area average mean values as in Eq. [6] (Color figure online).

dislocation arrangement parameter, $M$, by the CMWP procedure. The average distance between twin boundaries, $d_{\text {Twin }}$, can be obtained from $\beta$ as $d_{\text {Twin }}=\frac{a}{\sqrt{3}} \frac{1}{\beta}$, where it is assumed that twinning occurs along the $\{111\}$ planes and $a$ is the lattice constant of the sample. Figure 2(a) shows $\langle x\rangle_{\text {area }}, \rho$, and $\beta$ as a function of $\gamma$. The values of $\langle x\rangle_{\text {area }}, \rho, \mathbf{M}$, and $d_{\text {Twin }}$ are listed in Table I.

The $M$ value increases with $\gamma$ indicating that the dipole character of dislocations decreases. Figure 2(b) shows the log-normal size distribution function, $f(x)$, for the as-received and the HPT-deformed specimens determined by X-ray LPA and by dark-field TEM. The LPA size distribution corresponds to $\gamma \cong 15$. The area average mean crystallite or grain size values, $\langle x\rangle_{\text {area }}$, are shown by vertical arrows. The X-ray data indicate somewhat smaller grain size than dark-field TEM micrographs. This difference is most probably due to smallest grains

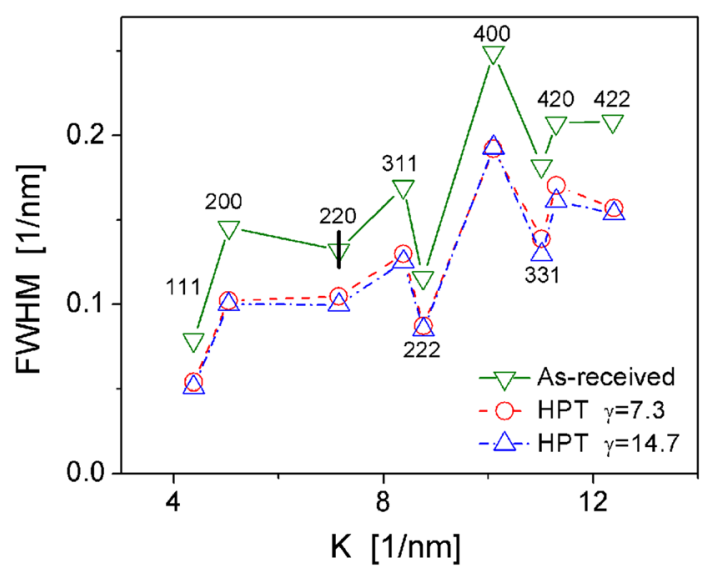

(a)

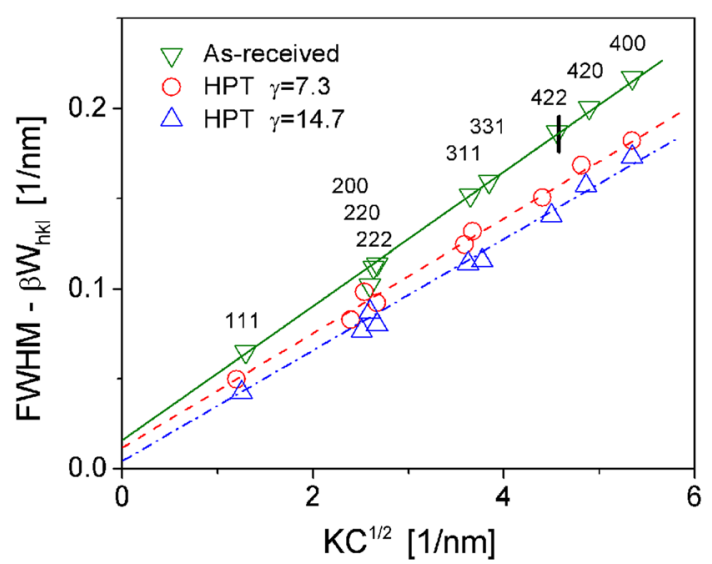

(b)

Fig. 3-FWHM values for the as-received and two deformed states of the specimen: FWHM values in the $(a)$ Williamson-Hall and $(b)$ modified Williamson-Hall plots. In latter, the FWHM values were corrected for twinning as in Ref. [15]. Errors of the FWHM values are given by the vertical black line.

not being counted in the TEM micrographs, whereas all coherent domains contribute to size broadening in the X-ray patterns.

\section{B. Qualitative Verification of Strain Line Broadening by the Williamson-Hall and Modified Williamson-Hall Methods}

The qualitative features of line broadening are best seen in the Williamson-Hall $(\mathrm{WH})^{[70]}$ and modified Williamson-Hall $(m \mathrm{WH})^{[20]}$ plots. In these plots, the FWHM of peaks are plotted either $v s K=2 \sin \theta / \lambda$ or $K \sqrt{\bar{C}}$, respectively, where $\theta$ and $\lambda$ are the diffraction angle and the wavelength of X-ray beam and $\bar{C}$ is the average dislocation contrast as provided by CMWP and defined in Eqs. [3] and [4]. The WH plots for the as-received and the HPT-deformed specimen measured at $0.33 R$ and $0.66 R$ shear strained to $\gamma=7.3$ and $\gamma=14.7$ are shown in Figure 3(a). The apparent weirdness of FWHM vs strain indicates strong strain anisotropy. In case there are no planar defects, strain anisotropy can be straightened in the $m \mathrm{WH}$ plot ${ }^{[20]}$ by 
replacing $\mathrm{K}$ with $\mathrm{K} \sqrt{\bar{C}}$. Planar defects introduce additional anisotropic broadening, the $h k l$ dependence of which is different from strain anisotropy. This additional broadening can be taken into account by correcting the measured FWHM values by the effect of planar defects ${ }^{[5,15,42]}$ :

$$
\mathrm{FWHM}^{*}=\mathrm{FWHM}-\beta W(h k l),
$$

where $\beta$ is the density of twin boundaries and $W(h k l)$ are $h k l$-dependent numbers characterizing broadening caused by twinning. ${ }^{[42]}$ The $m \mathrm{WH}$ plot corrected for twinning is shown in Figure 3(b). The figure shows a good linear relation between the FWHM values and $\mathrm{K} \sqrt{\bar{C}}$. The slopes of the linear regressions of the $0.33 R$ and $0.66 R$ FWHMs are smaller than that of the as-received specimen and are equal to each other, indicating a decrease of microstrain compared to the as-received state. The smallest intersection of the regression at $K=0$ corresponds to the $0.66 R$ position, indicating the largest size of coherently scattering domains. The qualitative trends revealed by the $\mathrm{mWH}$ plots in Figure 3(b) are in good correlation with the quantitative results listed in Table I. We note, however, that the breadth data can only provide qualitative information about the microstructure and should not be used for quantitative analysis. ${ }^{[29,30,71]}$

\section{Electron Microscopy Results of the Nanocrystalline Au-10 Pct Pd Samples}

The microstructures of the as-received and HPT-deformed samples were characterized by TEM shown in Figure 4. Figures 4(a), (b), (d), and (e) show typical bright-field and corresponding dark-field TEM micrographs of the as-received and HPT-deformed material, respectively. The micrographs indicate grain coarsening during HPT. The grain size distributions were obtained by evaluating more than 1000 grains from at least 10 dark-field TEM images well spread in the whole TEM samples. The histograms of grain size distribution of the as-received and HPT-deformed samples are shown in Figures 4(c) and (f), respectively. The histograms were fitted with log-normal size distribution functions according to Eq. [4]. The TEM-determined median, $m$, and variance, $\sigma$, of the as-received and HPT-deformed samples are $m_{\text {as-rec }}=13.2, \quad \sigma_{\text {as-rec }}=0.33$, and $m_{\mathrm{HPT}}=22.6, \sigma_{\mathrm{HPT}}=0.37$. The comparison of the TEM and X-ray determined $m$ and $\sigma$ values is shown in Table II indicating a reasonable correlation provided by
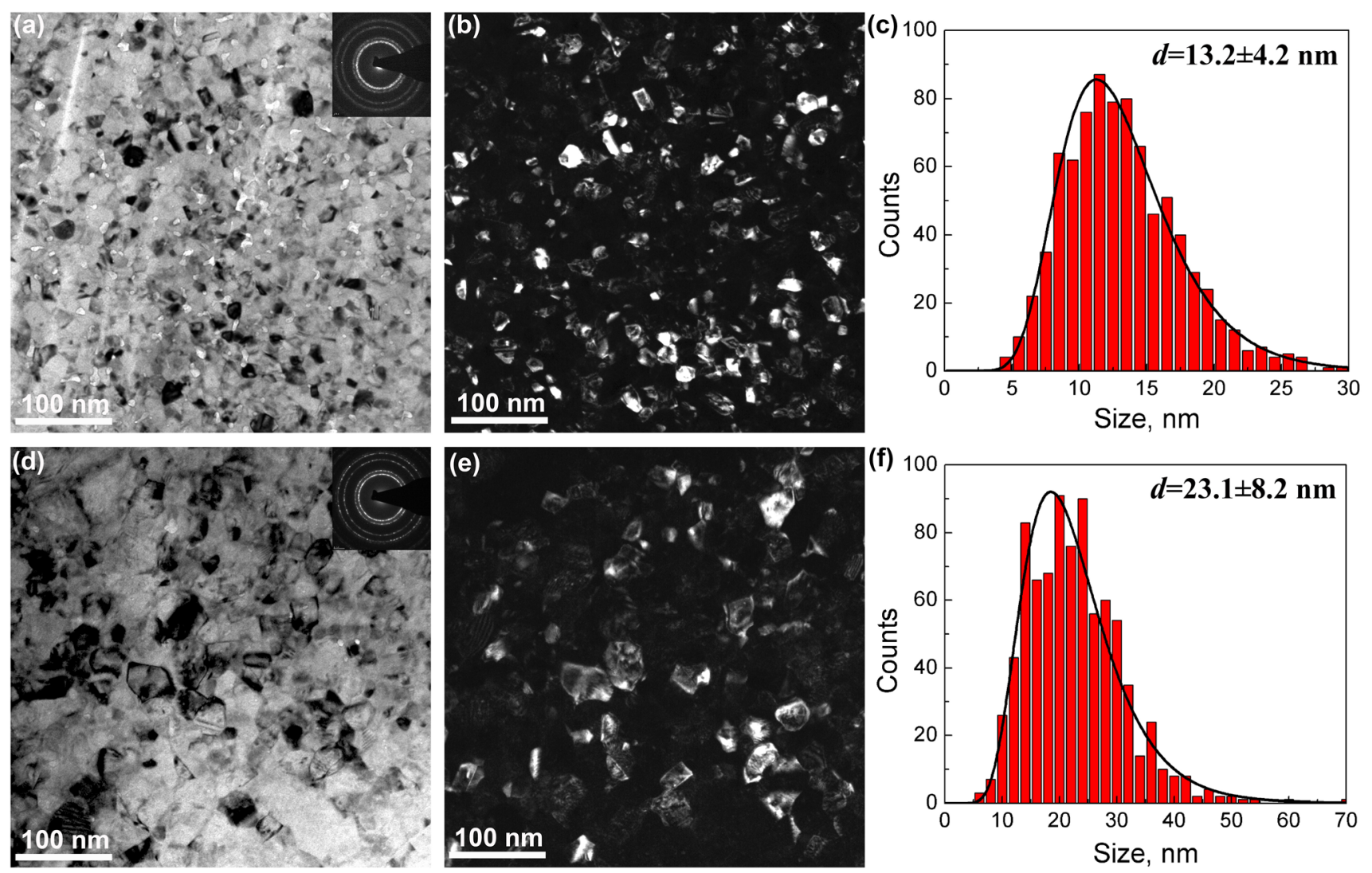

Fig. $4-(a, b)$ are bright-field and dark-field TEM images and $(c)$ a histogram showing the grain size distribution of about 1000 grains obtained from about 10 similar dark-field images of the as-received sample. $(d$, e) are bright-field and dark-field TEM images and $(f)$ a histogram showing the grain size distribution of about 1000 grains obtained from about 10 similar dark-field images of the HPT-deformed sample. 
Table II. Comparison of the X-ray and TEM Grain Size and Grain Size Distribution Data

\begin{tabular}{|c|c|c|c|c|c|c|}
\hline & \multicolumn{3}{|c|}{ X-ray LPA } & \multicolumn{3}{|c|}{ Dark-Field TEM } \\
\hline & $m(\mathrm{~nm})$ & $\sigma$ & $\langle x\rangle_{\text {area }}(\mathrm{nm})$ & $m(\mathrm{~nm})$ & $\sigma$ & $\langle x\rangle_{\text {area }}(\mathrm{nm})$ \\
\hline As-Received & $8.4( \pm 0.5)$ & $0.49( \pm 0.02)$ & $15.3( \pm 2)$ & $12.5( \pm 0.3)$ & $0.33( \pm 0.016)$ & $16.4( \pm 0.8)$ \\
\hline HPT & $12( \pm 0.8)$ & $0.44( \pm 0.1)$ & $19.5( \pm 5)$ & $21.1( \pm 0.5)$ & $0.37( \pm 0.05)$ & $29.5( \pm 1.7)$ \\
\hline
\end{tabular}

The median and variance, $m$ and $\sigma_{\mathrm{LN}}$, of the log-normal size distribution function and the area average mean crystallite and grain size of the as-received and HPT-deformed specimens determined by LPA and TEM, respectively. The X-ray LPA numbers of the HPT-deformed specimen are the averages of the HPT data listed in Table I.

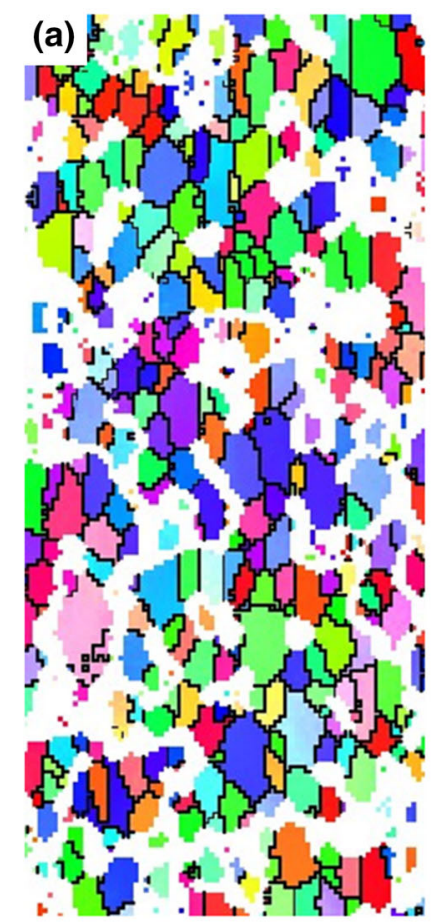

(e)

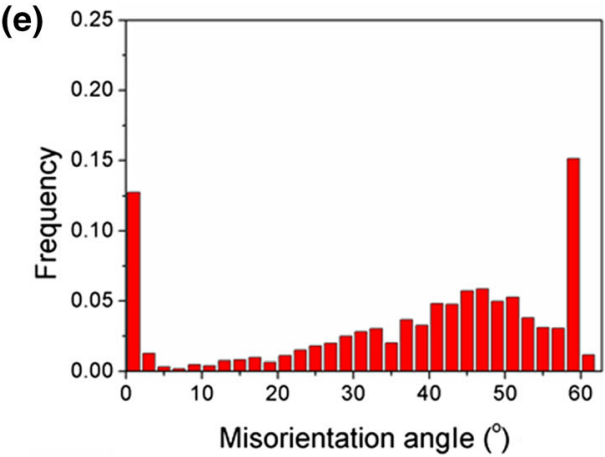

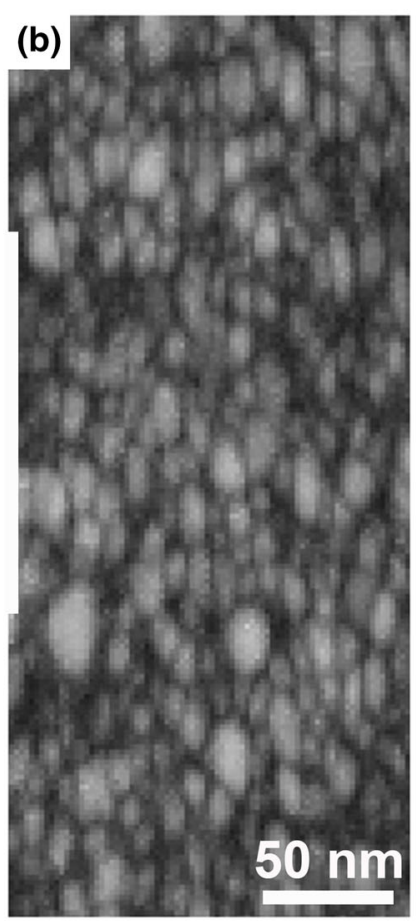
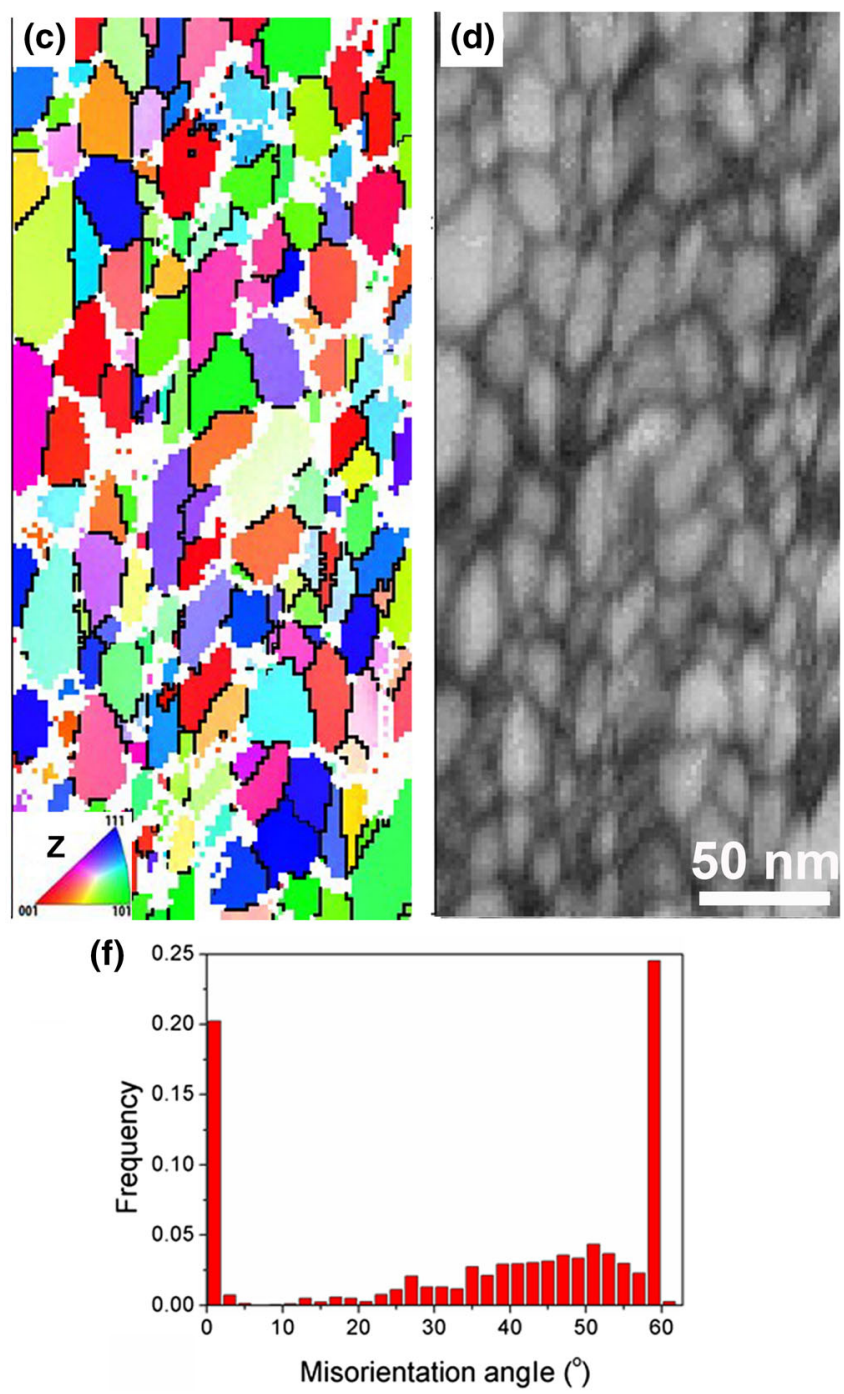

Fig. 5-Microstructural information obtained by TKD. $(a, c)$ Orientation maps, $(b, d)$ band contrast images of the as-received and HPT-deformed specimens. $(e, f)$ misorientation distribution histograms of the as-received and HPT-deformed samples, respectively.

the two different methods. As mentioned in paragraph 3.1 , the X-ray grain size values are slightly smaller than the TEM values most probably due to the smallest grains not being counted in the TEM micrographs.

Orientation maps of the grain structure are determined by Transmission Kikuchi Diffraction (TKD) shown for the as-received and the HPT-deformed samples in Figures 5(a) and (d), respectively. The corresponding band contrast images are shown in Figures 5(b) and (d). The orientation distributions in Figures 5(c) and (f) show that there are a large number of grains with low-angle GBs. Because of the limited angular resolution of the TKD technique, misorientations less than about $1 \mathrm{deg}$ were not considered in the 


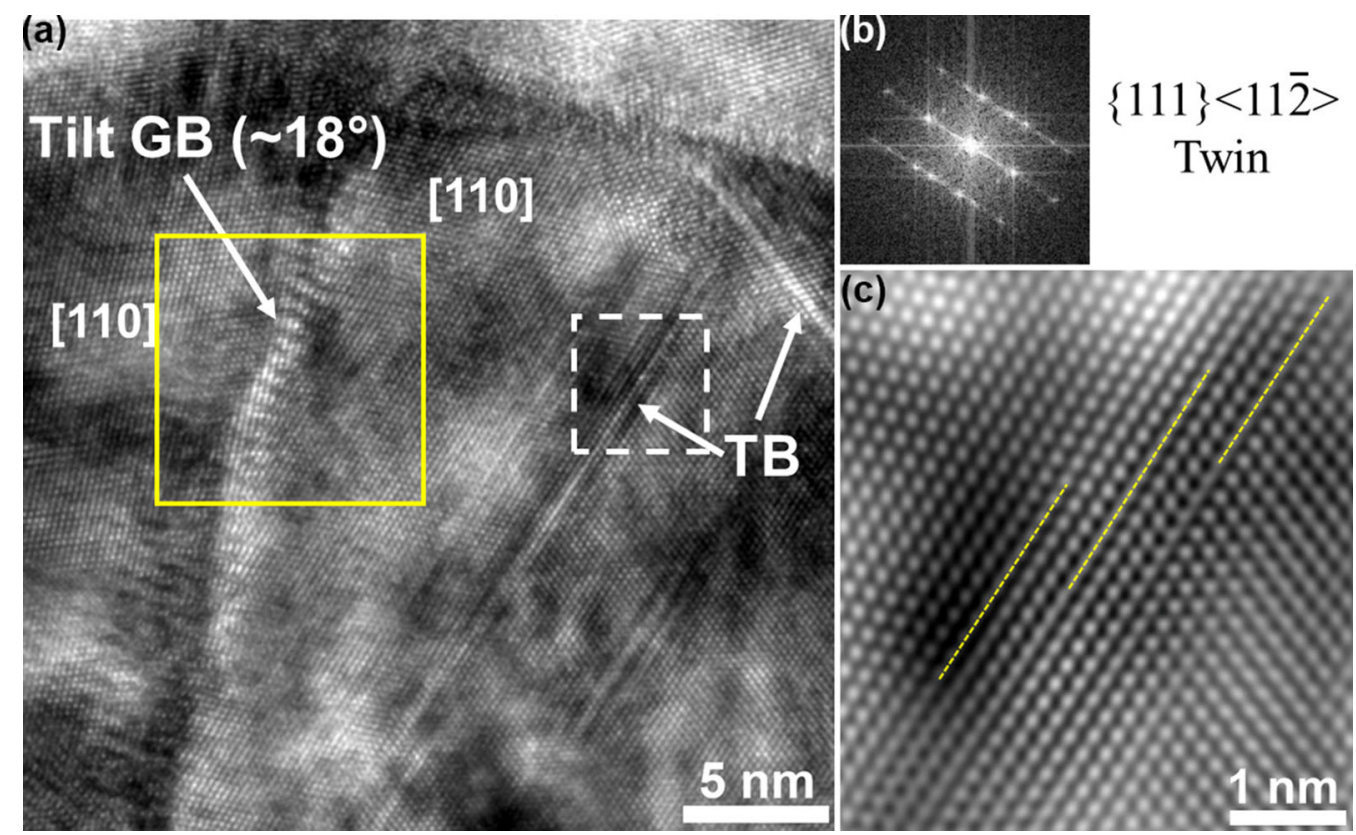

Fig. 6-HRTEM analysis of the sample after HPT deformation. (a) A HRTEM image showing a tilt grain boundary (GB) with tilt axis along the [110] direction and some twin boundaries (TB), $(b)$ is the FFT pattern of the yellow-framed region in (a), $(c)$ is the enlarged IFFT image of the white dash-line-framed region with $\{111\}\langle 11-2\rangle$ twins. The twin boundaries (TB) are marked by white arrows in (a) (Color figure online).
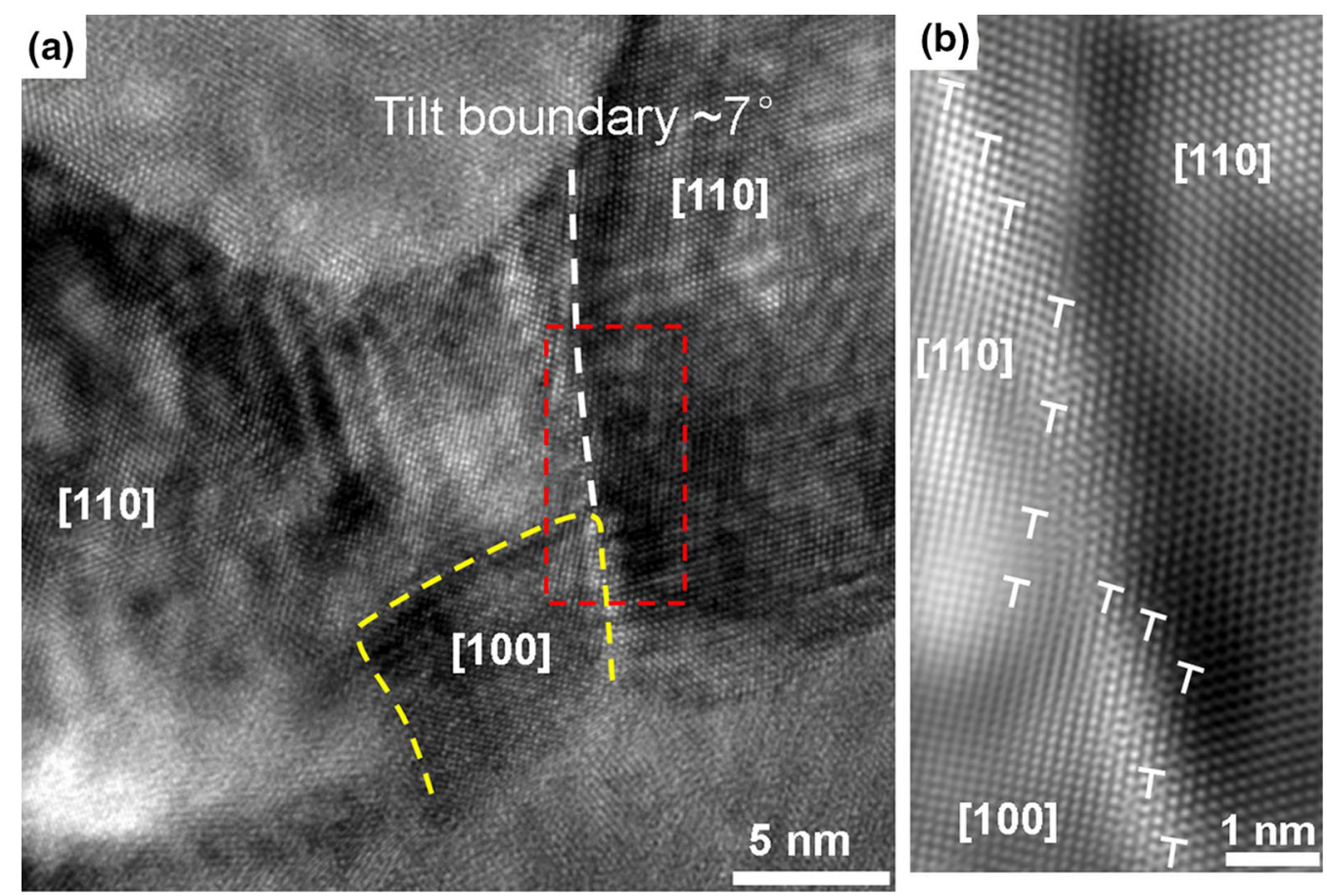

Fig. 7- (a) HRTEM image showing a region with a 7 deg tilt grain boundary and high-angle grain boundaries between [110] and [100] oriented grains with respect to the incident electron beam, $(b)$ an enlarged image from the red dash rectangle-framed region. The dislocations are indicated by $\mathrm{T}$ shape symbols (Color figure online).

misorientation distribution analysis. The misorientation peak around $60 \mathrm{deg}$ indicates twinning in correlation with X-ray observations shown in Figure 2(a) and Table I. The twins are identified as $\{111\}\langle 11-2\rangle$ twins by HRTEM shown in Figure 6.
Dislocations are identified in the boundaries of grains by HRTEM images in Figures 7 and 8 . Because of the very small grain size in the as-received specimen, HRTEM micrographs could only be obtained from the HPT-deformed specimens where the grains are 

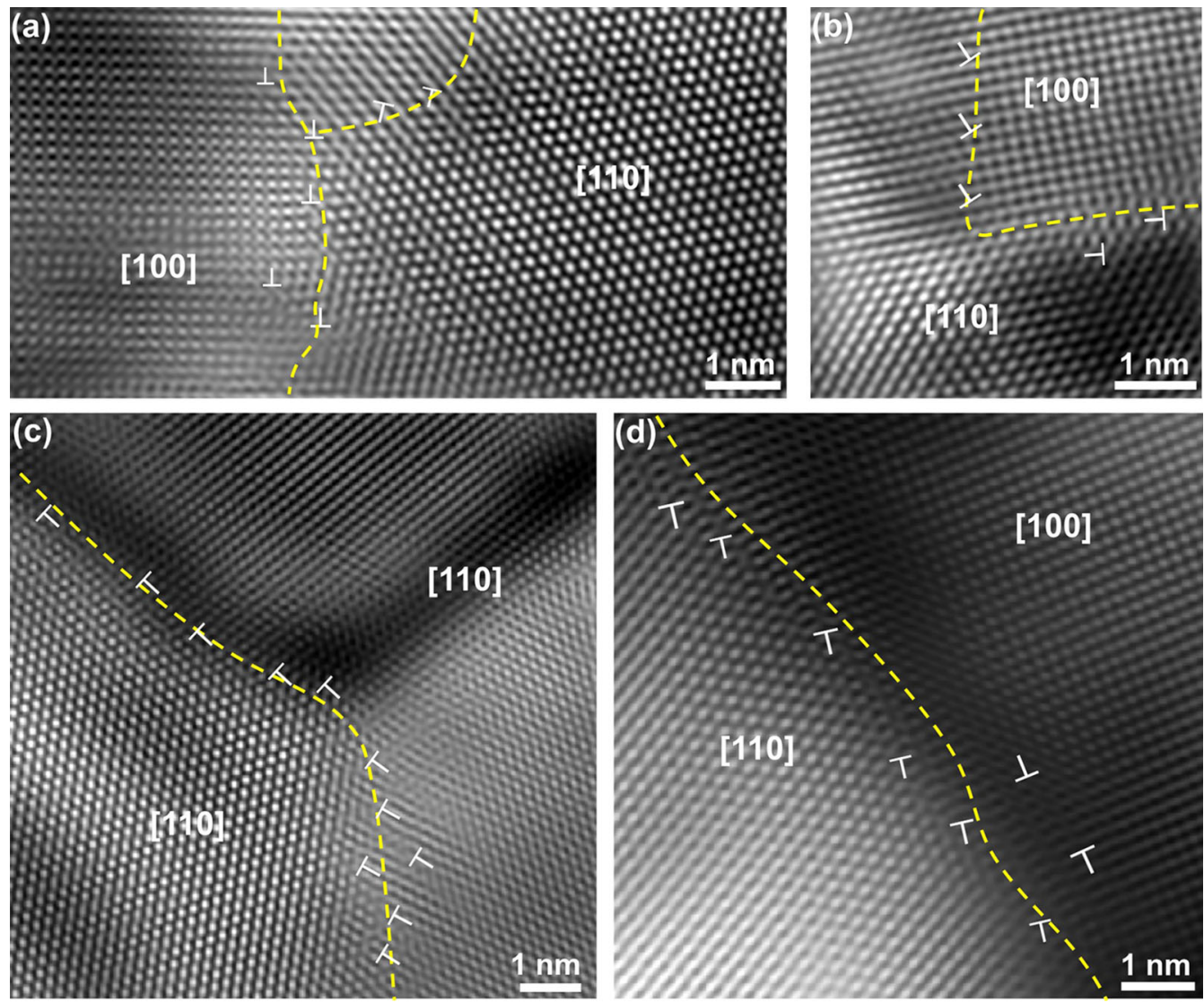

Fig. 8-HRTEM analysis of grain boundaries in the HPT-deformed sample: $(a)$ to $(d)$ are IFFT images of enlarged regions in HRTEM images of grain boundaries with grains oriented along [110] and [100] directions. Lattice dislocations in the grain boundaries are indicated by $\mathrm{T}$ shape symbols. It is noted that several more similar images were taken throughout the entire electron-transparent region of the sample.

somewhat larger. Figure 7(a) shows a 7 deg tilt boundary (white dash line), and a high-angle grain boundary between the [110] and [100] oriented grains (yellow dash-line-encircled region) with respect to the incident electron beam. The red dash-dot line-encircled region is enlarged in Figure $7 \mathrm{~b}$ where the dislocations in the tilt boundary and in the high-angle boundary are highlighted by white $\mathrm{T}$ signs.

The main issue in the present work, as mentioned before, is to find the source of strain causing strain broadening in X-ray line profiles in nanocrystalline materials. Our HRTEM micrographs provide direct evidence that dislocations in the present nanocrystalline alloys are within the grain boundary regions rather than in the grain interiors. In Figure 7(a), there is a 7 deg tilt boundary and there are several grain boundaries with high misorientations in Figure 7(b) and in Figure 8. Figures 8 (a) to (d) show four typical grain boundaries between [110] and [100] oriented grains. Lattice dislocations in the grain boundaries in all four micrographs are indicated by $\mathrm{T}$ shape symbols. We note here that many more similar images were taken throughout the entire electron-transparent region of the HPT-deformed specimen. Figure 9(a) shows a HRTEM micrograph of a single grain with incident electron beam along [110] zone axis. The corresponding inverse Fourier transform (IFFT) image is shown in Figure 9(b). The white dash line marks the grain boundary. The grain diameter is about $22( \pm 3) \mathrm{nm}$ and the grain interior is free from dislocations. The average dislocation distances in the boundaries in Figures 7 and 8 are about $1.5( \pm 1) \mathrm{nm}$. Taking the average grain size in the HPT-deformed state as $27( \pm 10) \mathrm{nm}$, the average dislocation density can be estimated to be between $1 \times 10^{16}$ and $5 \times 10^{16} \mathrm{~m}^{-2}$. This relatively large error margin is the consequence of the relatively wide grain size distribution, as shown in Figure 4, and of the fact that not all grain boundaries contain lattice dislocations, as it will be shown and discussed below in paragraphs 3.4. It should be noted that Moiré fringes are frequently observed in HRTEM images of nanocrystalline materials due to double diffraction in overlapping crystals through the foil thickness. Rentenberger et al. ${ }^{[72]}$ have pointed out that Moire fringes can erroneously be interpreted as regions with high density of dislocations. Figures 7 and 8 show clearly that there are no Moire fringes in the present HRTEM micrographs.

\section{Molecular Dynamic Simulations of Nanocrystalline $A u$ and Pd with Detailed Grain Structure Where Lattice Dislocations are Identified}

The samples used model completely random grain boundaries in a nanocrystalline material. In this sense, they do not model exactly the experimental material but 

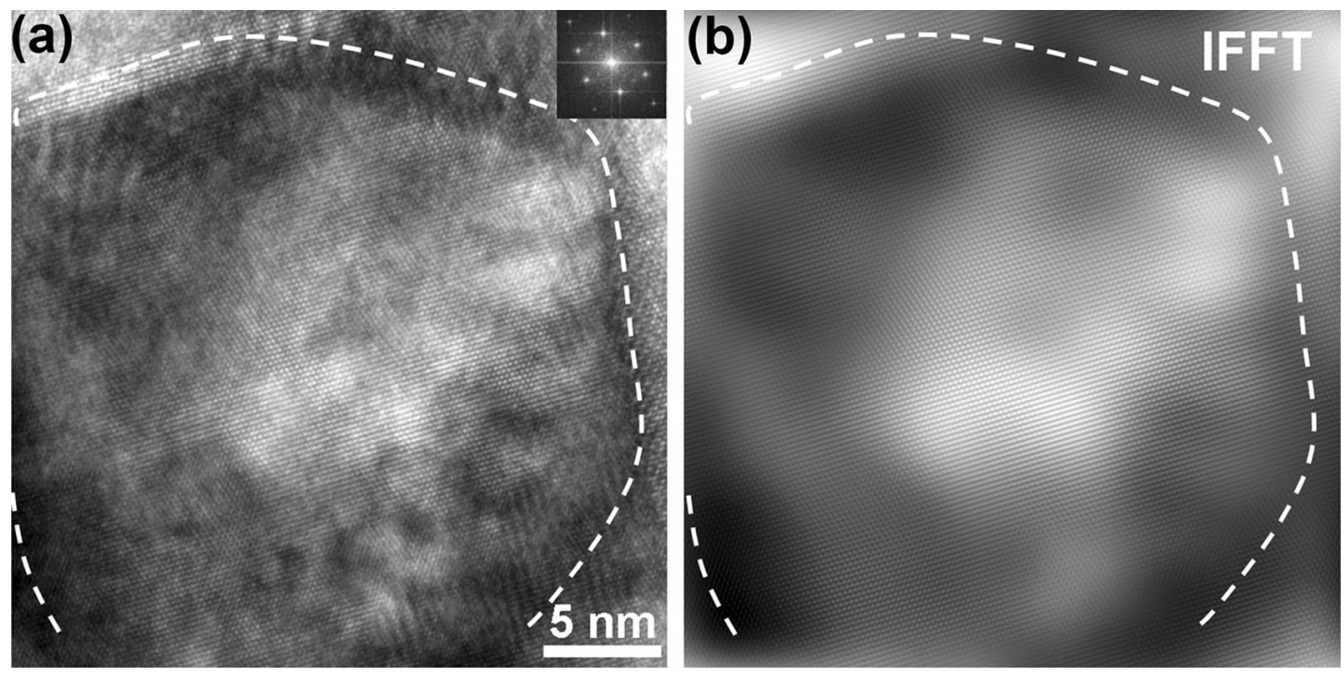

Fig. 9-Microstructure of a grain with a size of $\sim 26 \mathrm{~nm}$ in the sample after HPT: (a) HRTEM image, the corresponding FFT pattern (inset upright corner) indicates electron beam is along the [110] zone axis of this grain; $(b)$ the corresponding IFFT image of image (a).

represent a reasonable approximation to a typical random grain boundary. Similarly, because we use empirical potentials, the interactions do not represent exactly the experimental material. We are interested in general features that do not depend strongly on the details of the potential used. For this reason, we have used a pure Pd potential and we have also repeated the simulations with a pure $\mathrm{Au}$ potential to make sure the general trends are independent of the details of the potential.

The overall microstructure for the Pd sample is shown in Figure 10(a). About 79 pct of the atoms were identified as $f c c$ atom using the common neighbor analysis formalism as implemented in OVITO. ${ }^{[68]}$ These are shown in green in Figure 10(a). The remaining 21 pct of the atoms, in blue, are the atoms comprising the grain boundaries. The results for the sample of Au were very similar. After the relaxation procedure, the samples were analyzed for dislocation content using the Dislocation Extraction Algorithm (DXA). ${ }^{[68]}$ This procedure generates a geometric description of dislocation lines contained in an arbitrary crystalline model structure. Burgers vectors are determined reliably, and the extracted dislocation network fulfills the Burgers vector conservation rule at each node. DXA detects dislocations by iteratively constructing Burgers circuits to identify dislocation cores. The DXA implementation in OVITO extracts perfect lattice dislocations of the FCC lattice and partial dislocations in FCC crystals. In the present work, we have only utilized it to detect perfect dislocations in order to better match the experimental procedure, which detects perfect dislocations. In the construction of the Burgers circuits, the procedure requires two parameters. These parameters are called "trial circuit length" and "circuit stretchability." We have utilized the default values of 14 atom-to-atom steps for the trial circuit length and 9 atom-to-atom steps for the circuit stretchability.

Only perfect lattice dislocations of the $f c c$ lattice were detected in this procedure. They could be dislocations of the lattices of any of the grains comprising the grain boundary. Grain boundary dislocations or partial dislocations were not detected by this procedure. As expected for a relaxed sample under no applied stress, no dislocations were detected inside the grains. This is in agreement with the experimental results showing no dislocations inside the grains. Most interestingly, it was found that many of the grain boundaries in the sample contained a dislocation network as part of the structure. Figures 10(b) and (c) show two different sections of the sample illustrating that many of the boundaries have perfect lattice dislocations as part of their structure. Figures 10(d) and (e) show two examples of the detailed dislocation structure found in the nanocrystalline grain boundaries. The dislocation segments are colored according to dislocation character, from blue for edge to red for screw. While it is widely recognized that the structure of low-angle boundaries may be lattice dislocations, our results show that there are dislocations in the high-angle random grain boundaries. The average length of the dislocation segments is about 1.2 nanometers. A total of about 300 dislocations were detected in each sample, which corresponds to a volumetric dislocation density of about $4.7( \pm 0.5) \times 10^{16} \mathrm{~m}^{-2}$. This number arises from the large fraction of grain boundary material present in the nanocrystalline sample, and occurs despite the fact that there are no dislocations within the grains. The average planar dislocation density in the grain boundaries was found to be about $4 \times 10^{8} \mathrm{~m}^{-1}$. In recent work using a Ni potential and a similar technique, it was found that a large fraction of the boundaries studied contained significant densities of dislocations as part of their structure. ${ }^{[54]}$ 


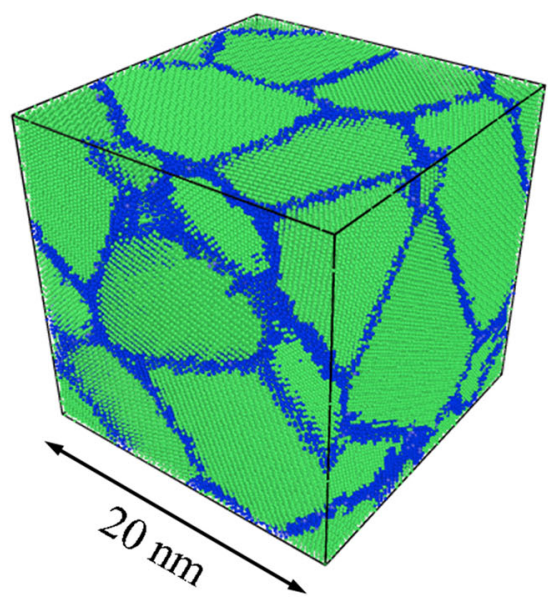

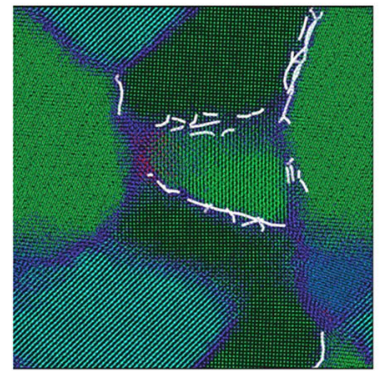

(b)

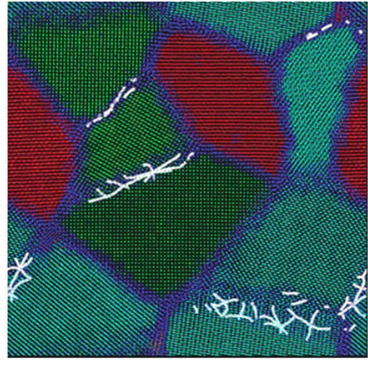

(c)

(a)

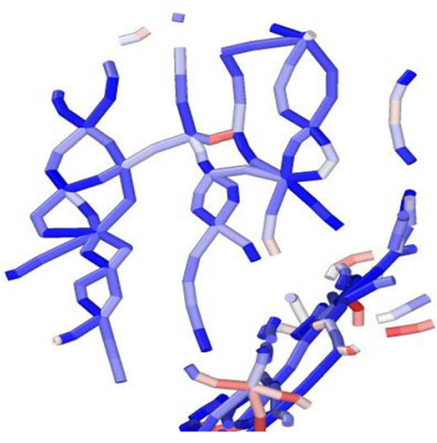

(d)

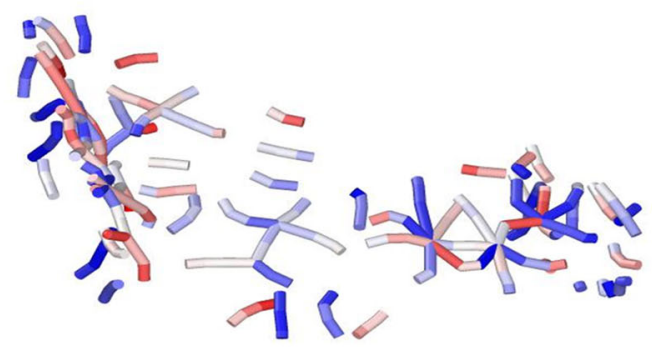

(e)

Fig. 10 - Results of the MD simulation: (a) resulting structure for the MD-simulated Pd sample. $(b, c)$ are two different sections of the sample illustrating that many of the boundaries have perfect dislocations as part of their structure. $(d, e)$ show two examples of the detailed dislocation structure in the nanocrystalline grain boundaries. Dislocation segments with perfect lattice Burgers vectors viewed close to perpendicular to the grain boundary plane. Coloring is according to dislocation character, from blue for edge to red for screw (Color figure online).

Table III. Values of the Average Grain Size and Average Dislocation Densities in the HPT-Deformed Pd-10 At. Pct Au Specimen Determined by X-ray LPA, Dark-Field TEM, and MD-Simulated Pd Nanocrystal

\begin{tabular}{llcc}
\hline Sample & \multicolumn{1}{c}{ Method } & Average Grain Size $(\mathrm{nm})$ & ${\text { Average Dislocation Density }\left(10^{16} \mathrm{~m}^{-2}\right)}^{2}$ \\
\hline HPT-Deformed Pd-10 At. Pct Au & XLPA & $19.5( \pm 5)$ & $1.32( \pm 0.3)$ \\
MD-Simulated Pd & HRTEM & $27( \pm 10)$ & $3( \pm 2)$ \\
\hline
\end{tabular}

For the sake of a better overview, the average grain size (crystallite size), $\langle\mathrm{x}\rangle_{\text {area }}$, and dislocation density, $\rho$, values obtained by X-ray diffraction, HRTEM, and MD simulations are compiled in Table III. As discussed at the end of paragraph 2.3.1, since in the present case the grain size is well below $100 \mathrm{~nm}$, the crystallite size, $\langle\mathrm{x}\rangle_{\text {area }}$, is identical with the grain size.

\section{DISCUSSION}

\section{A. Strain Distribution in the Near GB Region}

Based on MD simulations, Stukowski et al ${ }^{\left[{ }^{[6]}\right.}$ conclude that local displacements near GBs correlate over short distances insignificantly affecting the broadening of Bragg reflections. Instead, strain broadening arises from long-range displacement fields extending far from GBs, the origin of which is, however, not understood. In the present paragraph, we scrutinize the local lattice distortions near GBs deduced from MD simulations of nanocrystalline Pd shown in Fig. 4 in Reference 6. We show that, contrary to the assumptions in Reference 6, these lattice distortions are of long-range character following the strain function of Wilkens ${ }^{[23]}$ for dislocations. Stukowski et al. ${ }^{[6]}$ determined the average local distortion, $\bar{\delta}=\left[1 / 3\left(\varepsilon_{1}^{2}+\varepsilon_{2}^{2}+\varepsilon_{3}^{2}\right)\right]^{1 / 2}$, in MD-simulated nanocrystalline $\mathrm{Pd}$, where $\varepsilon_{1}, \varepsilon_{2}$, and $\varepsilon_{3}$ are the relative variation of the local lattice parameter in the three 
principle directions of the Green strain tensor. More details are in paragraph 2.2 .2 in Reference 6. The average distortion $v s$ the distance of GBs in the MD-simulated nanocrystalline $\mathrm{Pd}$ of $9.2 \mathrm{~nm}$ grain size is shown in Fig. 4 of Reference 6. The histograms show that the average distortion is large close to the GB and decays towards the grain interior region. Virtual X-ray diffraction patterns were also produced and the squareroot of the mean square strain, $\sqrt{\left\langle\varepsilon^{2}\right\rangle}(m \mathrm{WH})$, was determined from the modified Williamson-Hall plot. This value is denoted as $\varepsilon_{X R D}$ in Fig. 4 in Reference 6. The atomic displacements and the corresponding stresses are shown as colored figures for a cross section of one of the MD-simulated nanocrystalline Pd sample in Fig. 3 in Reference 6. The atomic positions in the GB regions were not investigated. One of the histograms of Fig. 4 in Reference 6 and the digitized values are shown in Figures 11(a) and (b), respectively. The analysis indicates that the digitized distortion follows the Krivoglaz-Wilkens strain function, $f(\eta)$, shown as a blue curve in Figure 11(b), where $\eta$ is the distance from GBs in $R_{\mathrm{e}}$ units. The colored figure of local stresses in Figure 11(c) shows that the elevated stresses appear pairwise on the opposite sides of the GBs. Encouraged by this observation, the local distortion distribution is plotted in Figure 11(d) as appearing symmetrically on both sides of a grain boundary intruding into the two neighboring grains. There has been an attempt to interpret strain broadening in the diffraction patterns of nanograin materials as enhanced Debye-Waller factors in the GB regions. ${ }^{[6,9]}$ The strain field of the enhanced Debye-Waller factor would, however, decrease as $1 / x^{2}$, where $x$ is the distance from GBs. The same digitized values shown in Figure 11(d) (open ochre circles) are shown in double logarithmic scale in Figure 11(e) along with the Krivoglaz-Wilkens strain function (blue curve) and a $1 / x^{2}$ function (dashed straight line). The figure shows that the average strain decays substantially slower than $1 / x^{2}$ and it follows the strain distribution function typical for dislocations. The strain distribution maps of Stukowski et al. in Reference 6 are in good correlation with the present X-ray, HRTEM, TKD, and MD simulation results which prove the presence of lattice dislocations in GBs and GB regions. A very recent $\mathrm{MD}$ simulation work has also proved the presence of lattice dislocations in GBs and GB regions. ${ }^{[54]}$

The value of $\varepsilon_{X R D}$ in Fig. 4 in Reference 6 is much smaller than the distortion distribution shown as histograms. Although the slopes of modified Williamson-Hall plots, corrected for dislocation contrast, are in correlation with dislocation densities, $c f$. References 5, 15, and 20, the concrete values of dislocation densities cannot be determined from such slopes. $^{[23,29,30,73,74]}$ A large number of investigations proved that the breadths and shape of line profiles depend on the coupled values of the number density, $\rho$, and the effective outer cut-off radius, $R_{\mathrm{e}}$, of dislocations. ${ }^{[23,29,30,38,39,41,43,47-51]}$ These two parameters determine the distortion distribution function, $f(\eta)$, derived by Wilkens. ${ }^{[23]}$ It is probably the first time that the distortion distribution function, $f(\eta)$, has been determined in an MD simulation 'experiment' in such a clear form as shown in Figure 4 in the work of Stukowski et al. ${ }^{[6]}$

\section{B. Dislocation Density as a Function of Grain Size}

The HRTEM and MD simulations show that a substantial fraction of GBs contain lattice dislocations with large densities. Keeping in mind that below a certain threshold of grain size there are no dislocations in the grain interiors, the following model of dislocation density $v s$ grain size is suggested. The model is using the finding of Swygenhoven et al. ${ }^{[75]}$ who found that GBs in nanocrystalline and coarse grain materials have very similar structures. Based on this, we assume that the linear dislocation density in GBs, $\rho_{\mathrm{IF}}$, does not depend on grain size (the subscript IF refers to interface and note that the unit of $\rho_{\mathrm{IF}}$ is reciprocal length.) The volume dislocation density corresponding to $\mathrm{GBs}$, smeared over entire grains, $\rho_{G B}^{v o l}$, can be written as

$$
\rho_{G B}^{v o l}=\alpha \frac{\rho_{\mathrm{IF}}}{D}
$$

where $\mathrm{D}$ is the average grain size and $\alpha$ is a constant. As discussed before, grain interiors are free from dislocations when the grain size is below a certain threshold value, $D_{\text {thr. }}$. A simple exponential function is suggested to give the dislocation density in grain interiors, $\rho_{\mathrm{GI}}$, as a function of grain size:

$$
\rho_{\mathrm{GI}}=\rho_{\mathrm{CG}}\left[1-\exp \left(-\kappa \mathrm{D}^{\mathrm{n}}\right)\right],
$$

where $\rho_{\mathrm{CG}}$ is the average dislocation density when grain size is large or coarse, $\kappa$ and $n$ are constants, adjusted to give the grain size threshold, $D_{\text {thr }}$ (below which the grain interiors are free from dislocations). When the grain size is smaller than $D_{\mathrm{thr}}$, i.e., $D \leq D_{\mathrm{thr}}$, the total dislocation density is provided by the dislocations in the GB region, i.e., $\rho_{G B}^{v o l}$. However, at larger grain size, when $D \geq D_{\mathrm{thr}}, \rho_{G B}^{v o l}$ becomes negligible compared to $\rho_{\mathrm{GI}}$. Since $\rho_{G B}^{v o l}$ and $\rho_{\mathrm{GI}}$ are dominant in two distinct grain size regions, the total dislocation density, $\rho$, can be given, approximately, as the sum of the two dislocation densities:

$$
\rho_{\mathrm{GI}}+\rho_{G B}^{v o l}=\rho_{\mathrm{CG}}\left[1-\exp \left(-\kappa \mathrm{D}^{\mathrm{n}}\right)\right]+\alpha \frac{\rho_{\mathrm{IF}}}{\mathrm{D}} .
$$

Equation [11] is shown in Figure 12. The solid black line is the dislocation density in the grain interiors, $\rho_{\mathrm{GI}}$, the black dash line is the volume dislocation density in the GBs, $\rho_{G B}^{v o l}$, and the blue dash-dot line is the total dislocation density, $\rho$. The vertical dot arrow indicates the grain size threshold, $D_{\mathrm{thr}}$, below which grain interiors are free from dislocations. The open red circles are the dislocation densities in the $\mathrm{Au}-10$ pct $\mathrm{Pd}$ specimen provided by CMWP, shown in Figure 2(a) and listed in Table I. The constants in Eq. [11] were adjusted to match $\rho$ in Eq. [11] with the measured dislocation density values: $\alpha=4, \quad \rho_{\mathrm{CG}}=1.25 \times$ $10^{16} \mathrm{~m}^{-2}, \kappa=2.8 \times 10^{-5}\left[D^{-n}\right]$, and $n=4$. The threshold of grain size, $D_{\text {thr }}$, below which grain interiors 
(a)
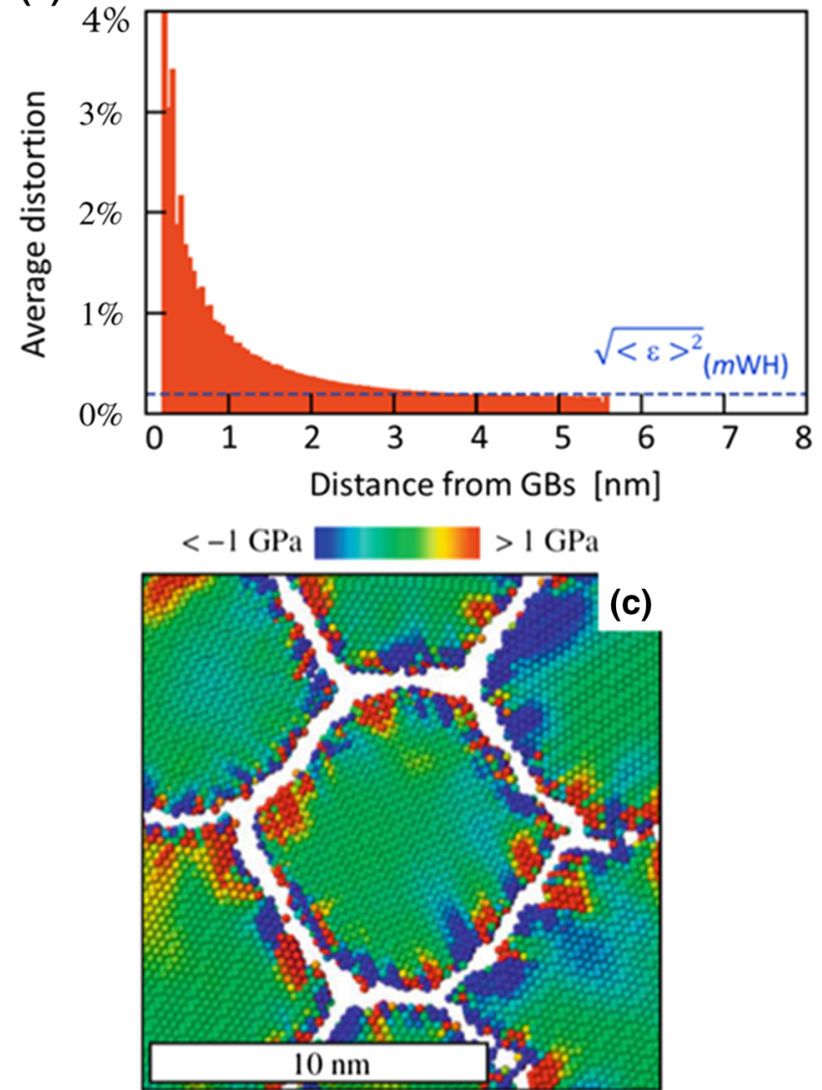

(b)

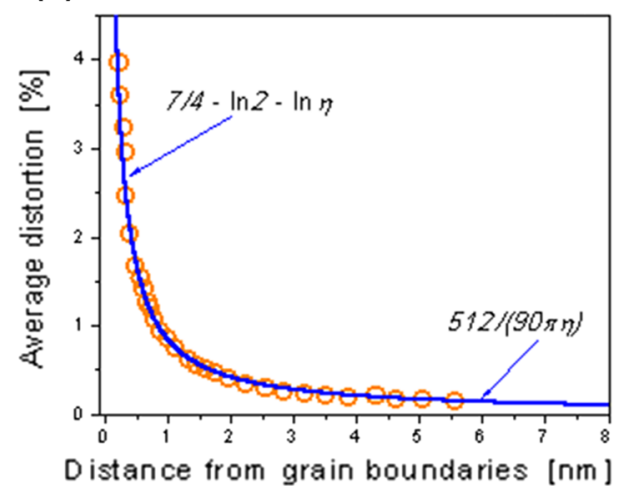

(d)

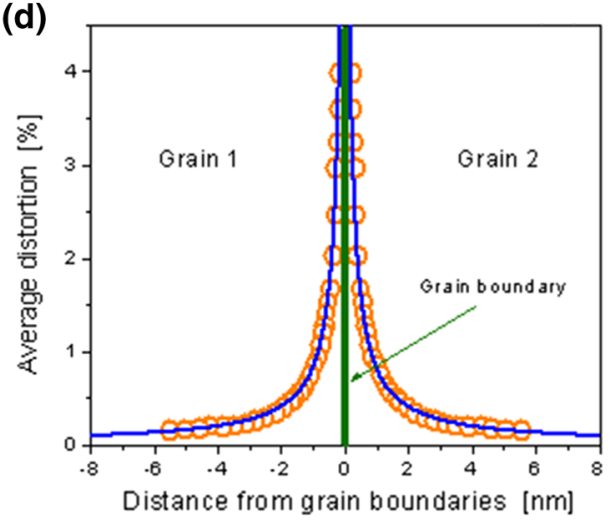

(e)

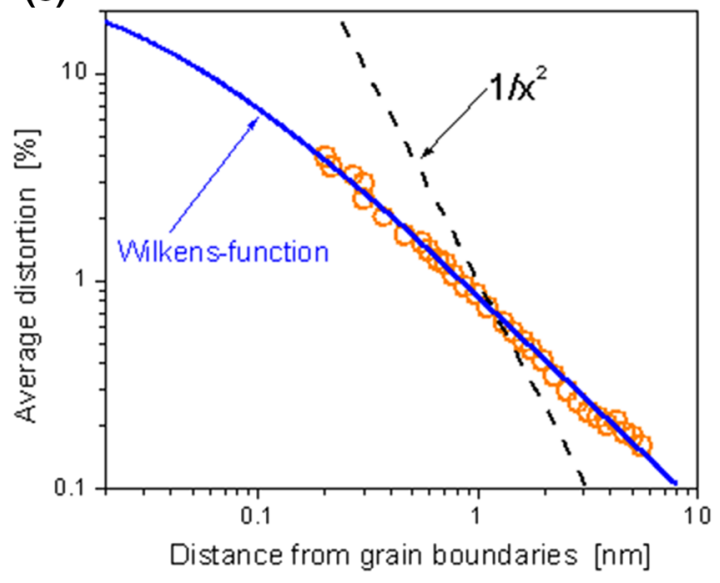

Fig. 11-(a) Average distortion distribution vs the distance from GBs in nanocrystalline Pd determined by MD simulation. The basis of the figure was borrowed by courtesy from Ref. [6]. (b) Digitized values (open ochre circles) of the distortion values in (a) and the Krivoglaz-Wilkens strain function $f(\eta)$ (blue curve) (see Eq. [2] and Eq. (A8) in Ref. [23]) $v s$ the distance from GBs as in (a). $\eta$ in the $f(\eta)$ function is scaled in units of $R_{\mathrm{e}}$. (c) Stress fields in a cross section of one of MD-simulated nanocrystalline Pd with grain size of $9.2 \mathrm{~nm}$. Atoms are colored for the hydrostatic stress component. Parts of the figures here were borrowed from Ref. [6] by courtesy of the authors. (d) Average distortion vs the distance from GBs in MD-simulated nanocrystalline Pd on the two sides of a GB (vertical green line). (e) Digitized values (open ochre circles) as in (b) with the Krivoglaz-Wilkens strain function (blue curve) and a $1 / x^{2}$ function (dashed straight line) in double logarithmic scale, where $x$ is the distance from grain boundaries (Color figure online).

become free from dislocations, was taken to be $D_{\mathrm{thr}} \cong 13 \mathrm{~nm} \cdot{ }^{[3,4]}$ Figure 12 does not show the as-received $\rho$ value since this corresponds to sample preparation and is irrelevant for the deformation process. The dip in the total dislocation density at very small grain sizes might be related to the inverse Hall-Petch behavior of nanocrystalline metals. ${ }^{[1]}$ We note that a more rigorous model would have to take into account the 


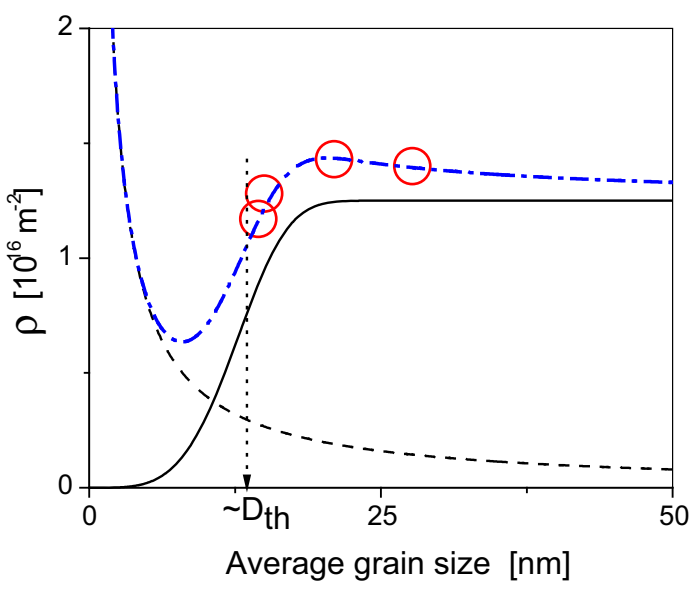

Fig. 12-Semischematic image of the dislocation density vs the average grain size, according to Eq. [3]. The decaying dash line is the total dislocation density in grain boundaries. The solid black line is the total dislocation density in grain interiors assuming that below a grain size threshold of about $D_{\mathrm{thr}}$ the grain interiors become free of dislocations. The dash-dot blue line is the sum of the dash and solid lines. The open red circles are the dislocation densities measured by $\mathrm{X}$-ray line profile analysis (Color figure online).

volume fractions of GBs and grain interior regions. This is, however, beyond the scope of the present work.

In elastically anisotropic polycrystalline materials, elastic compatibility strains and stresses (ECSs) can build up between grains of different orientations. ${ }^{[76]}$ As soon as the local ECSs reach a critical value, misfit dislocations will emanate in the GB regions reducing these strains and stresses. ${ }^{[55-58]}$ In Reference 54, the types and Burgers vectors of dislocations were determined by MD simulation in an $f c c$ nanocrystalline specimen. It was shown that the overwhelming majority of dislocations prevail in the GB regions and that almost all dislocations along one particular GB have the same Burgers vector (see Figure 8 in Reference 54). These are the misfit dislocations reducing the ECSs. Since the Burgers vectors along a particular GB are the same, we can assume that these dislocations are similar to GNDs in the gradient model of plasticity. ${ }^{[5,77-80]}$ The strain fields of these misfit dislocations are of long-range character in good correlation with the strain distribution determined by Stukowski in Reference 6 and discussed in the previous paragraph.

\section{CONCLUSIONS}

We carried out X-ray line profile analysis, TEM, HRTEM, and TKD experiments on inert gas-condensed Pd-10 at. pct $\mathrm{Au}$ nanocrystalline specimens in the as-received and HPT-deformed states. The experiments are supported by MD simulations of nanocrystalline $\mathrm{Pd}$ and $\mathrm{Au}$ specimens consisting of 12 grains of an average diameter of $7.5 \mathrm{~nm}$. The goal of the work has been to find the source of large strain broadening of X-ray diffraction peaks from nanocrystalline materials. The HRTEM experiments and the MD simulations reveal that there are large dislocation densities in the GB regions. The method of DXA was implemented in OVITO extracts of the MD-simulated specimens. The procedure revealed perfect lattice dislocations of the $f c c$ lattice and partial dislocations in $f c c$ crystals all clustering within the GB regions. Dislocation densities have been assessed from several HRTEM micrographs of the deformed specimen and determined by the DXA method in the undeformed MD-simulated Pd crystal. These values are $3( \pm 2) \times 10^{16} \mathrm{~m}^{-2}$ and $4.7( \pm 0.5) \times 10^{16}$ $\mathrm{m}^{-2}$, respectively. X-ray line broadening gave $1.32( \pm 0.3) \times 10^{16} \mathrm{~m}^{-2}$ in good correlation with the other two values, nonetheless as a lower bound of those.

Spatial distribution of distortions stemming from GB regions was determined by Stukowski et al. ${ }^{[6]}$ in an MD-simulated Pd crystal. We have shown that it follows the Wilkens strain function ${ }^{[23]}$ typical for strain distributions produced by dislocations. The faster, $1 / x^{2}$ type strain distribution, typical for random displacement of atoms, is not supported by the experimental or MD simulation evidences.

Assuming that grain boundary structures in nanocrystalline and coarse grain materials are very similar, a schematic model is suggested for the dislocation density as a function of grain size. We suggest that the dislocation density in the GB regions is almost like a material constant depending mostly on the misorientation and structure of GBs. The model shows, on the one hand, that when the grain size is smaller than about $20 \mathrm{~nm}$ and the grain interior regions become more-orless free from dislocations the average dislocation density in the crystal can still be substantially large. At these small grain size values, the volume fraction of GBs becomes significant and the dislocation density in GBs becomes dominant in the entire crystal. On the other hand, even in coarse grain polycrystals, the GB regions do consist of substantial dislocation densities playing an important role in the plastic deformation of materials.

\section{ACKNOWLEDGMENTS}

G.R. gratefully acknowledges the support of the János Bolyai Research Fellowship of the Hungarian Academy of Sciences. T.U. is grateful for the EPSRC Leadership Fellowship [EP/I005420/1] for the study of dislocation structures in metallic materials by X-ray diffraction. The authors are grateful to Dr A. Stukowski for private communication about DXA application in GB regions and for his kind permission to use Figures 11(a) and (c).

\section{OPEN ACCESS}

This article is distributed under the terms of the Creative Commons Attribution 4.0 International License (http://creativecommons.org/licenses/by/4.0/), which permits unrestricted use, distribution, and reproduction in any medium, provided you give appropriate credit to the original author(s) and the 
source, provide a link to the Creative Commons license, and indicate if changes were made.

\section{REFERENCES}

1. A.H. Chokshi, A. Rosen, J. Karch, and H. Gleiter: Scripta Metall., 1989, vol. 23, pp. 1679-83.

2. V. Yamakov, D. Wolf, S.R. Phillpot, A.K. Mukherjee, and H. Gleiter: Nat. Mater., 2002, vol. 1, pp. 45-48.

3. H. Van Swygenhoven and J.R. Weertman: Mater. Today, 2006, vol. 9, pp. 24-31

4. J. Markmann, V. Yamakov, and J. Weissmuller: Scripta Mater., 2008, vol. 59, pp. 15-18

5. W. Skrotzki, A. Eschke, B. Jóni, T. Ungár, L.S. Tóth, Yu. Ivanisenko, and L. Kurmanaeva: Acta Mater., 2013, vol. 61, pp. 7271-84.

6. A. Stukowski, J. Markmann, J. Weissmuller, and K. Albe: Acta Mater., 2009, vol. 57, pp. 1648-54.

7. J. Markmann, D. Bachurin, L. Shao, P. Gumbsch, and J. Weissmuller: Eur. Phys. Lett., 2010, vol. 89, p. 66002.

8. A. Leonardi, K.R. Beyerlein, T. Xu, M. Li, M. Leoni, and P. Scardi: Z. Kristallogr. Proc., 2011, vol. 1, pp. 37-42.

9. P. Scardi, L. Rebuffi, M. Abdellatief, A. Flora, and A. Leonardi: J. Appl. Crystallogr., 2017, vol. 50, pp. 508-18.

10. Y.T. Zhu, X.Z. Liao, and X.L. Wu: Prog. Mater. Sci., 2012 vol. 57, pp. 1-62.

11. Y. Ivanisenko, L. Kurmanaeva, J. Weissmueller, K. Yang, J. Markmann, H. Rosner, T. Scherer, and H.J. Fecht: Acta Mater., 2009, vol. 57, pp. 3391-3401

12. H. Van Swygenhoven, P.M. Derlet, and A.G. Froseth: Nat. Mater., 2004, vol. 3, pp. 399-403.

13. K. Zhang, J.R. Weertman, and J.A. Eastman: Appl. Phys. Lett., 2004, vol. 85, pp. 5197-99.

14. J. Schafer, A. Stukowski, and K. Albe: Acta Mater., 2011, vol. 59 pp. 2957-68.

15. T. Ungár, S. Ott, P.G. Sanders, A. Borbély, and J.R. Weertman: Acta Mater., 1998, vol. 46, pp. 3693-99.

16. T. Ungár, L. Li, G. Tichy, W. Pantleon, H. Choo, and P.K. Liaw: Scripta Mater., 2011, vol. 64, pp. 876-79.

17. L. Li, T. Ungár, L.S. Toth, W. Skrotzki, Y.D. Wang, Y. Ren, H. Choo, Z. Fogarassy, X.T. Zhou, and P.K. Liaw: Metall. Mater. Trans. A, 2016, vol. 47A, pp. 6632-44.

18. C.E. Krill and R. Birringer: Philos. Mag. A, 1998, vol. 77 pp. $621-40$.

19. H. Van Swygenhoven, B. Schmitt, P. M. Derlet, S. Van Petegem, A. Cervellino, Z. Budrovic, S. Brandstetter, A. Bollhalder, and M. Schild: Rev. Sci. Instrum., 2006, vol. 77, p. 013902.

20. T. Ungár and A. Borbély: Appl. Phys. Lett., 1996, vol. 69, pp. 3173-75.

21. M.A. Krivoglaz and K.P. Rjaboshapka: Fiz. Met. Metalloved., 1963, vol. 15, pp. 18-31.

22. M.A. Krivoglaz: in X-ray and Neutron Diffraction in Nonideal Crystals, Springer, Berlin, 1996.

23. M. Wilkens: in Fundamental Aspects of Dislocation Theory, Special Publication No. 317, J.A. Simmons, R. de Wit, and R. Bullough, eds., U.S. National Bureau of Standards, Washington, DC, 1970 , vol. II, pp. 1195-1221.

24. Y. Ivanisenko, E.D. Tabachnikova, I. Psaruk, S.N. Smirnov, A. Kilmametov, A. Kobler, C. Kubel, L. Kurmanaeva, K. Csach, Y. Mishkuf, T. Scherer, Y.A. Semerenko, and H. Hahn: Int. J. Plasticity, 2014, vol. 60, pp. 40-57.

25. T. Inami, S. Okuda, H. Maeta, and H. Ohtsuka: Mater. Trans. JIM, 1998, vol. 39, pp. 1029-32.

26. T. Ungár, I. Dragomir, A. Revesz, and A. Borbély: J. Appl. Crystallogr., 1999, vol. 32, pp. 992-1002.

27. M. Wilkens and K. Eckert: Z. Physik., 1963, vol. 170, pp. 459-70.

28. A. Guinier and F. Sebbileau: C. R. Acad. Sci. Paris, 1952, vol. 235, pp. $888-90$.

29. G. Ribárik and T. Ungár: Mater. Sci. Eng. A, 2010, vol. 528, pp. 112-21.

30. T. Ungár, L. Balogh, and G. Ribárik: Metall. Mater. Trans. A, 2010, vol. 41A, pp. 1202-09.

31. E.F. Bertaut: Acta Crystallogr., 1950, vol. 3, pp. 14-18.
32. L. Balogh, G. Ribárik, and T. Ungár: J. Appl. Phys., 2006, vol. 100, p. 023512.

33. L. Balogh, G. Tichy, and T. Ungár: J. Appl. Crystallogr., 2009, vol. 42, pp. 580-91.

34. E. Estevez-Rams, A. Penton-Madrigal, R. Lora-Serrano, and J. Martinez-Garcia: J. Appl. Crystallogr., 2001, vol. 34, pp. 730-36.

35. E. Estevez-Rams, B. Aragon-Fernandez, H. Fuess, and A. Penton-Madrigal: Phys. Rev. B, 2003, vol. 68, p. 064111.

36. E. Estevez-Rams, M. Leoni, P. Scardi, B. Aragon-Fernandez, and H. Fuess: Philos. Mag., 2003, vol. 83, pp. 4045-57.

37. M.M.J. Treacy, J.M. Newsam, and M.W. Deem: Proc. R. Soc. Lond. Ser. A, 1991, vol. 433, pp. 499-520.

38. G. Ribárik, B. Jóni, and T. Ungár: Mater. Sci. Technol., 2019, vol. 35 , pp. 1508-14.

39. T. Ungár, G. Tichy, J. Gubicza, and R.J. Hellmig: Powder Diffract., 2005, vol. 20, pp. 366-75.

40. J.I. Langford, A. Boultif, J.P. Auffredic, and D. Louer: J. Appl. Crystallogr., 1993, vol. 26, pp. 22-33.

41. P. Scardi and M. Leoni: Acta Crystallogr. A, 2001, vol. 57, pp. 604-13.

42. B.E. Warren: Prog. Met. Phys., 1959, vol. 8, pp. 147-202.

43. I. Groma, T. Ungár, and M. Wilkens: J. Appl. Crystallogr., 1988, vol. 21 , pp. $47-53$.

44. J.E. Bailey: Philos. Mag., 1963, vol. 86, pp. 223-36.

45. M. Wilkens: Acta Metall., 1967, vol. 15, pp. 1412-15.

46. U.F. Kocks: Acta Metall., 1967, vol. 15, pp. 1415-17.

47. M. Wilkens, K. Herz, and H. Mughrabi: Z. Metallde., 1980, vol. 71, pp. 376-84.

48. T. Ungár, H. Mughrabi, D. Ronnpagel, and M. Wilkens: Acta Metall., 1984, vol. 32, pp. 333-42.

49. R. Kuzel and P. Klimanek: J. Appl. Crystallogr., 1989, vol. 22, pp. 299-307.

50. A. Borbély, I. Dragomir-Cernatescu, G. Ribárik, and T. Ungár: $J$. Appl. Crystallogr., 2003, vol. 36, pp. 160-62.

51. M. Leoni, J. Martinez-Garcia, and P. Scardi: J. Appl. Crystallogr., 2007, vol. 40, pp. 719-24.

52. I.C. Dragomir and T. Ungár: J. Appl. Crystallogr., 2002, vol. 35, pp. 556-64.

53. T. Ungár and G. Tichy: Phys. Status Solidi A, 1999, vol. 171, pp. 425-34.

54. B. Kuhr and D. Farkas: Model. Simul. Mater. Sci. Eng., 2019, vol. 27, p. 045005

55. M.F. Ashby: Philos. Mag. A, 1970, vol. 21, pp. 399-424.

56. A.J. Wilkinson, T.B. Britton, J. Jiang, and P.S. Karamched: IOP Conf. Ser. Mater. Sci. Eng., 2014, vol. 55, p. 012020.

57. D. Fullwood, B. Adams, J. Basinger, T. Ruggles, A. Khosravani, C. Sorensen, and J. Kacher: in Diffuse Scattering and the Fundamental Properties of Materials, R. Barabash, G.E. Ice, and P.E.A. Turchi, eds., Imperial College Press, London, pp. 405-37.

58. D. Lunt, A. Orozco-Caballero, R. Thomas, P. Honniball, P. Frankel, M. Preuss, and J. Quinta da Fonseca: Mater. Charact., 2018, vol. 139, pp. 355-63.

59. H. Mughrabi, T. Ungár, W. Kienle, and M. Wilkens: Philos. Mag. $A$, 1986, vol. 53, pp. 793-813.

60. H.A. Kuhn, H. Biermann, T. Ungár, and H. Mughrabi: Acta Metall. Mater., 1991, vol. 39, pp. 2783-94.

61. T. Ungár, S. Harjo, T. Kawasaki, Y. Tomota, G. Ribárik, and Z. Shi: Metall. Mater. Trans. A, 2017, vol. 48A, pp. 159-67.

62. M. Knezevic, H.F. Al-Harbi, and S.R. Kalidindi: Acta Mater., 2009, vol. 57, pp. 1777-84.

63. H. Abdolvand, J. Wright, and A.J. Wilkinson: Nat. Commun., 2018, vol. 9, art. no. 171. https://doi.org/10.1038/s41467-01702213-9.

64. K. Huang and H.H. Wills: Proc. Roy. Soc. A Math. Phys., 1947, vol. 190, pp. 102-17.

65. D. Farkas: Curr. Opin. Solid State Mater. Sci., 2013, vol. 17, pp. 284-97.

66. S.M. Foiles, M.I. Baskes, and M.S. Daw: Phys. Rev. B, 1986, vol. 33, pp. 7983-91.

67. S. Plimpton: J. Comput. Phys., 1995, vol. 117, pp. 1-19

68. A. Stukowski: Model. Simul. Mater. Sci., 2009, vol. 18, p. 015012.

69. A. Stukowski and K. Albe: Model. Simul. Mater. Sci., 2010, vol. 18 , p. 085001 
70. G.K. Williamson and W.H. Hall: Acta Metall., 1953, vol. 1, pp. 22-31.

71. P. Scardi, M. Leoni, and R. Delhez: J. Appl. Crystallogr., 2004, vol. 37, pp. 381-90.

72. C. Rentenberger, T. Waitz, and H.P. Karnthaler: Scripta Mater., 2004, vol. 51, pp. 789-94.

73. G. Zilahi, T. Ungár, and G. Tichy: J. Appl. Crystallogr., 2015, vol. 48, pp. 418-30.

74. I. Groma: in Mesoscale Models: From Micro-Physics to Macro-Interpretation, S. Mesarovic, F. Samuel, and Z. Hussein, eds., Springer, Cham, 2019, pp. 87-139.72.

75. H. Van Swygenhoven, A. Caro, and D. Farkas: Mater. Sci. Eng. A, 2001, vols. 309-310, pp. 440-44.
76. E. Macherauch: in Application of Fracture Mechanics to Materials and Structures, G.C. Sih, E. Sommer, and W. Dahl, eds., Springer, Dordrecht, 1984. pp. 157-92.

77. A.S. Aargon and P. Haasen, Acta Metall. Mater., 1993, vol. 41, pp. 3289-3306.

78. N.A. Fleck, G.M. Muller, M.F. Ashby, and J.W. Hutchinson: Acta Metall. Mater., 1994, vol. 42, pp. 475-87.

79. H. Mughrabi: Philos. Mag., 2006, vol. 86, pp. 4037-54.

80. L.S. Toth, C.F. Gu, B. Beausir, J.J. Fundenberger, and M. Hoffman: Acta Mater., 2016, vol. 117, pp. 35-42.

Publisher's Note Springer Nature remains neutral with regard to jurisdictional claims in published maps and institutional affiliations. 